\title{
Traveling Wave Solutions of a Generalized Camassa-Holm Equation: A Dynamical System Approach
}

\author{
Lina Zhang and Tao Song \\ Department of Mathematics, Huzhou University, Huzhou, Zhejiang 313000, China \\ Correspondence should be addressed to Lina Zhang; zsdzln@126.com
}

Received 1 August 2015; Accepted 14 September 2015

Academic Editor: Maria Gandarias

Copyright (C) 2015 L. Zhang and T. Song. This is an open access article distributed under the Creative Commons Attribution License, which permits unrestricted use, distribution, and reproduction in any medium, provided the original work is properly cited.

\begin{abstract}
We investigate a generalized Camassa-Holm equation $C(3,2,2): u_{t}+k u_{x}+\gamma_{1} u_{x x t}+\gamma_{2}\left(u^{3}\right)_{x}+\gamma_{3} u_{x}\left(u^{2}\right)_{x x}+\gamma_{3} u\left(u^{2}\right)_{x x x}=0$. We show that the $C(3,2,2)$ equation can be reduced to a planar polynomial differential system by transformation of variables. We treat the planar polynomial differential system by the dynamical systems theory and present a phase space analysis of their singular points. Two singular straight lines are found in the associated topological vector field. Moreover, the peakon, peakon-like, cuspon, smooth soliton solutions of the generalized Camassa-Holm equation under inhomogeneous boundary condition are obtained. The parametric conditions of existence of the single peak soliton solutions are given by using the phase portrait analytical technique. Asymptotic analysis and numerical simulations are provided for single peak soliton, kink wave, and kink compacton solutions of the $C(3,2,2)$ equation.
\end{abstract}

\section{Introduction}

Mathematical modeling of dynamical systems processing in a great variety of natural phenomena usually leads to nonlinear partial differential equations (PDEs). There is a special class of solutions for nonlinear PDEs that are of considerable interest, namely, the traveling wave solutions. Such a wave may be localized or periodic, which propagates at constant speed without changing its shape.

Many powerful methods have been presented for finding the traveling wave solutions, such as the Bäcklund transformation [1], tanh-coth method [2], bilinear method [3], symbolic computation method [4], and Lie group analysis method [5]. Furthermore, a great amount of works focused on various extensions and applications of the methods in order to simplify the calculation procedure. The basic idea of those methods is that, by introducing different types of Ansatz, the original PDEs can be transformed into a set of algebraic equations. Balancing the same order of the Ansatz then yields explicit expressions for the PDE waves. However, not all of the special forms for the PDE waves can be derived by those methods. In order to obtain all possible forms of the PDE waves and analyze qualitative behaviors of solutions, the bifurcation theory plays a very important role in studying the evolution of wave patterns with variation of parameters [6-9].

To study the traveling wave solutions of a nonlinear PDE

$$
\Phi\left(u, u_{t}, u_{x}, u_{x x}, u_{x t}, u_{t t}, \ldots\right)=0,
$$

let $\xi=x-c t$ and $u(x, t)=\varphi(\xi)$, where $c$ is the wave speed. Substituting them into (1) leads the PDE to the following ordinary differential equation:

$$
\Phi_{1}\left(\varphi, \varphi^{\prime}, \varphi^{\prime \prime}, \ldots\right)=0 .
$$

Here, we consider the case of (2) which can be reduced to the following planar dynamical system:

$$
\begin{aligned}
& \frac{d \varphi}{d \xi}=\varphi^{\prime}=y, \\
& \frac{d y}{d \xi}=F(\varphi, y),
\end{aligned}
$$

through integrals. Equation (3) is called the traveling wave system of the nonlinear PDE (1). So, we just study the 
traveling wave system (3) to get the traveling wave solutions of the nonlinear PDE (1).

Let us begin with some well-known nonlinear wave equations. The first one is the Camassa-Holm $(\mathrm{CH})$ equation [10]

$$
u_{t}-u_{t x x}+3 u u_{x}=2 u_{x} u_{x x}+u u_{x x x}
$$

arising as a model for nonlinear waves in cylindrical axially symmetric hyperelastic rods, with $u(x, t)$ representing the radial stretch relative to a prestressed state where Camassa and Holm showed that (4) has a peakon of the form $u(x, t)=$ $c e^{-|x-c t|}$. Among the nonanalytic entities, the peakon, a soliton with a finite discontinuity in gradient at its crest, is perhaps the weakest nonanalyticity observable by the eye [11].

To understand the role of nonlinear dispersion in the formation of patters in liquid drop, Rosenau and Hyman [12] introduced and studied a family of fully nonlinear dispersion Korteweg-de Vries equations

$$
u_{t}+\left(u^{m}\right)_{x}+\left(u^{n}\right)_{x x x}=0 .
$$

This equation, denoted by $K(m, n)$, owns the property that, for certain $m$ and $n$, its solitary wave solutions have compact support [12]. That is, they identically vanish outside a finite core region. For instance, the $K(2,2)$ equation admits the following compacton solution:

$$
u(x, t)= \begin{cases}\frac{4 c}{3} \cos ^{2}\left(\frac{x-c t}{4}\right), & |x-c t| \leq 2 \pi \\ 0, & \text { otherwise. }\end{cases}
$$

The Camassa-Holm equation, the $K(2,2)$ equation, and almost all integrable dispersive equations have the same class of traveling wave systems which can be written in the following form [13]:

$$
\begin{aligned}
& \frac{d \varphi}{d \xi}=y=\frac{1}{D^{2}(\varphi)} \frac{\partial H}{\partial y} \\
& \frac{d y}{d \xi}=-\frac{1}{D^{2}(\varphi)} \frac{\partial H}{\partial \varphi}=-\frac{D^{\prime}(\varphi) y^{2}+g(\varphi)}{D^{2}(\varphi)}
\end{aligned}
$$

where $H=H(\varphi, y)=(1 / 2) y^{2} D^{2}(\varphi)+\int D(\varphi) g(\varphi) d \varphi$ is the first integral. It is easy to see that (4) is actually a special case of (3) with $F(\varphi, y)=-\left(1 / D^{2}(\varphi)\right)(\partial H / \partial \varphi)$. If there is a function $\varphi=\varphi_{s}$ such that $D\left(\varphi_{s}\right)=0$, then $\varphi=\varphi_{s}$ is a vertical straight line solution of the system

$$
\begin{aligned}
& \frac{d \varphi}{d \zeta}=y D(\varphi), \\
& \frac{d y}{d \zeta}=-D^{\prime}(\varphi) y^{2}-g(\varphi),
\end{aligned}
$$

where $d \xi=D(\varphi) d \zeta$ for $\varphi \neq \varphi_{s}$. The two systems have the same topological phase portraits except for the vertical straight line $\varphi=\varphi_{s}$ and the directions in time. Consequently, we can obtain bifurcation and smooth solutions of the nonlinear PDE (1) through studying the system (8), if the corresponding orbits are bounded and do not intersect with the vertical straight line $\varphi=\varphi_{s}$. However, the orbits, which do intersect with the vertical straight line $\varphi=\varphi_{s}$ or are unbounded but can approach the vertical straight line, correspond to the non-smooth singular traveling waves. It is worth of pointing out that traveling waves sometimes lose their smoothness during the propagation due to the existence of singular curves within the solution surfaces of the wave equation.

Most of these works are concentrated on the nonlinear wave equations with only a singular straight line [6-9]. But till now there have been few works on the integrable nonlinear equations with two singular straight lines or other types of singular curves [13-15].

In 2004, Tian and Yin [16] introduced the following fully nonlinear generalized Camassa-Holm equation $C(m, n, p)$ :

$$
\begin{aligned}
u_{t}+k u_{x}+\gamma_{1} u_{x x t}+\gamma_{2}\left(u^{m}\right)_{x}+\gamma_{3} u_{x}\left(u^{n}\right)_{x x} \\
+\gamma_{4} u\left(u^{p}\right)_{x x x}=0,
\end{aligned}
$$

where $k, \gamma_{1}, \gamma_{2}, \gamma_{3}$, and $\gamma_{4}$ are arbitrary real constants and $m$, $n$, and $p$ are positive integers. By using four direct ansatzs, they obtained kink compacton solutions, nonsymmetry compacton solutions, and solitary wave solutions for the $C(2,1,1)$ and $C(3,2,2)$ equations.

Generally, it is not an easy task to obtain a uniform analytic first integral of the corresponding traveling wave system of (9). In this paper, we consider the cases $m=3$, $n=p=2$, and $\gamma_{3}=\gamma_{4}$. Then, (9) reduces to the $C(3,2,2)$ equation

$$
\begin{gathered}
u_{t}+k u_{x}+\gamma_{1} u_{x x t}+\gamma_{2}\left(u^{3}\right)_{x}+\gamma_{3} u_{x}\left(u^{2}\right)_{x x} \\
+\gamma_{3} u\left(u^{2}\right)_{x x x}=0 .
\end{gathered}
$$

Actually, we have already considered a special $C(3,2,2)$ equation in [17], namely, $\gamma_{1}=-1, \gamma_{2}=-3$, and $\gamma_{3}=-1$, where the bifurcation of peakons are obtained by applying the qualitative theory of dynamical systems. In this work, a more general $C(3,2,2)$ equation (10) is studied. Different bifurcation curves are derived to divide the parameter space into different regions associated with different types of phase trajectories. Meanwhile, it is interesting to point out that the corresponding traveling wave system of (10) has two singular straight lines compared with (4), which therefore gives rise to a variety of nonanalytic traveling wave solutions, for instance, peakons, cuspons, compactons, kinks, and kink-compactons.

This paper is organized as follows. In Section 2, we analyze the bifurcation sets and phase portraits of corresponding traveling wave system. In Section 3, we classify single peak soliton solutions of (10) and give the parametric representations of the smooth soliton solutions, peakon-like solutions, cuspon solutions, and peakon solutions. In Section 4, we obtain the kink wave and kink compacton solutions of (10). A short conclusion is given in Section 5. 


\section{Bifurcation Sets and Phase Portraits}

In this section, we shall study all possible bifurcations and phase portraits of the vector fields defined by (10) in the parameter space. To achieve such a goal, let $u(x, t)=\varphi(\xi)$ with $\xi=x-c t$ be the solution of (10), then it follows that

$$
\begin{gathered}
(k-c) \varphi^{\prime}-\gamma_{1} c \varphi^{\prime \prime \prime}+\gamma_{2}\left(\varphi^{3}\right)^{\prime}+\gamma_{3} \varphi^{\prime}\left(\varphi^{2}\right)^{\prime \prime} \\
+\gamma_{3} \varphi\left(\varphi^{2}\right)^{\prime \prime \prime}=0,
\end{gathered}
$$

where $\varphi^{\prime}=\varphi_{\xi}, \varphi^{\prime \prime}=\varphi_{\xi \xi}$, and $\varphi^{\prime \prime \prime}=\varphi_{\xi \xi \xi}$. Integrating (11) once and setting the integration constant as $g$, we have

$$
(k-c) \varphi-\gamma_{1} c \varphi^{\prime \prime}+\gamma_{2} \varphi^{3}+\gamma_{3} \varphi\left(\varphi^{2}\right)^{\prime \prime}=-g .
$$

Clearly, (12) is equivalent to the planar system

$$
\begin{aligned}
& \frac{d \varphi}{d \xi}=y, \\
& \frac{d y}{d \xi}=\frac{\beta \varphi^{3}+\varphi y^{2}+\epsilon \varphi+\sigma}{\rho-\varphi^{2}},
\end{aligned}
$$

where $\rho=c \gamma_{1} / 2 \gamma_{3}, \beta=\gamma_{2} / 2 \gamma_{3}, \sigma=g / 2 \gamma_{3}$, and $\epsilon=(k-c) / 2 \gamma_{3}$ $\left(\gamma_{3} \neq 0\right)$. System (13) has the first integral

$$
H(\varphi, y)=\frac{\beta}{4} \varphi^{4}+\frac{1}{2} \epsilon \varphi^{2}+\sigma \varphi+\frac{1}{2} \varphi^{2} y^{2}-\frac{1}{2} \rho y^{2}=h .
$$

Obviously, for $\rho>0$, system (13) is a singular traveling wave system [14]. Such a system may possess complicated dynamical behavior and thus generate many new traveling wave solutions. Hence, we assume $\rho>0$ in the rest of this paper $\left(\rho=\varrho^{2}, \varrho>0\right)$. The phase portraits defined by the vector fields of system (13) determine all possible traveling wave solutions of (10). However, it is not convenient to directly investigate (13) since there exist two singular straight lines $\varphi=\varrho$ and $\varphi=-\varrho$ on the right-hand side of the second equation of (13). To avoid the singular lines temporarily, we define a new independent variable $\zeta$ by setting $(d \xi / d \zeta)=$ $\varrho^{2}-\varphi^{2}$; then, system (13) is changed to a Hamiltonian system, written as

$$
\begin{aligned}
& \frac{d \varphi}{d \zeta}=\left(\varrho^{2}-\varphi^{2}\right) y, \\
& \frac{d y}{d \zeta}=\varphi y^{2}+\beta \varphi^{3}+\epsilon \varphi+\sigma .
\end{aligned}
$$

System (15) has the same topological phase portraits as system (13) except for the singular lines $\varphi=\varrho$ and $\varphi=-\varrho$.

We now investigate the bifurcation of phase portraits of the system (15). Denote that

$$
f(\varphi)=\beta \varphi^{3}+\epsilon \varphi+\sigma .
$$

Let $M\left(\varphi_{e}, y_{e}\right)$ be the coefficient matrix of the linearized system of (15) at the equilibrium point $\left(\varphi_{e}, y_{e}\right)$; then,

$$
M\left(\varphi_{e}, y_{e}\right)=\left(\begin{array}{cc}
-2 \varphi_{e} y_{e} & y_{\epsilon}^{2}+f^{\prime}\left(\varphi_{e}\right) \\
\varrho^{2}-\varphi_{e}^{2} & 2 \varphi_{e} y_{e}
\end{array}\right),
$$

and at this equilibrium point, we have

$$
\begin{aligned}
J\left(\varphi_{e}, y_{e}\right) & =\operatorname{det} M\left(\varphi_{e}, y_{e}\right) \\
& =\left(\varphi_{e}^{2}-\varrho^{2}\right) f^{\prime}\left(\varphi_{e}\right)-\left(3 \varphi_{e}^{2}+\varrho^{2}\right) y_{e}^{2} .
\end{aligned}
$$

By the theory of planar dynamical systems, for an equilibrium point of a Hamiltonian system, if $J<0$, then it is a saddle point, a center point if $J>0$, and a degenerate equilibrium point if $J=0$.

From the above analysis, we can obtain the bifurcation curves and phase portraits under different parameter conditions.

Let

$$
\begin{aligned}
& \beta_{1}(\sigma)=-\frac{4 \epsilon^{3}}{27 \sigma^{2}}, \\
& \beta_{2}(\sigma)=\frac{\sigma-\epsilon \varrho}{\varrho^{3}}, \\
& \beta_{3}(\sigma)=\frac{-\sigma-\epsilon \varrho}{\varrho^{3}} .
\end{aligned}
$$

Clearly, for $\beta>\beta_{1}(\sigma)$, the function $f(\varphi)=0$ has three real roots $\varphi_{1}, \varphi_{2}$, and $\varphi_{3}\left(\varphi_{1}>\varphi_{2}>\varphi_{3}\right)$; that is, system (15) has three equilibrium points $E_{i}\left(\varphi_{i}, 0\right), i=1,2,3$ on the $\varphi$-axis. When $\beta<\beta_{3}(\sigma)$, (15) has two equilibrium points $S_{1,2}\left(\varrho, \pm Y_{1}\right)$ on the straight line $\varphi=\sqrt{a}$, where $Y_{1}=$ $\sqrt{\left(-\sigma-\varrho\left(\epsilon+\beta \varrho^{2}\right)\right) / \varrho}$. When $\beta<\beta_{2}(\sigma)$, system (15) has two equilibrium points $S_{3,4}\left(-\varrho, \pm Y_{2}\right)$ on the straight line $\varphi=-\varrho$, where $Y_{2}=\sqrt{\left(\sigma-\varrho\left(\epsilon+\beta \varrho^{2}\right)\right) / \varrho}$. Notice that on making the transformation $\sigma \rightarrow-\sigma, \varphi \rightarrow-\varphi, \zeta \rightarrow-\zeta$, system (15) is invariant. This means that, for $\sigma<0$, the phase portraits of (15) are just the reflections of the corresponding phase portraits of (15) in the case $\sigma>0$ with respect to the $y$-axis. Thus, we only need to consider the case $\sigma \geq 0$. To know the dynamical behavior of the orbits of system (15), we will discuss two cases: $\epsilon>0$ and $\epsilon<0$.

\subsection{Case I: $\epsilon>0$}

Lemma 1. Suppose that $\epsilon>0$. Denote $h_{\varrho}^{+}=H\left(\varrho, \pm Y_{1}\right), h_{\varrho}^{-}=$ $H\left(-\varrho, \pm Y_{2}\right)$, and $h_{i}=H\left(\varphi_{i}, 0\right), i=1,2,3$.

(1) For $\sigma>0$ and $\beta_{1}(\sigma)<\beta<\beta_{3}(\sigma)$, there exists one and only one curve $\beta=\beta_{4}(\sigma)$ on the $(\sigma, \beta)$-plane on which $h_{\varrho}^{+}=h_{2}$; for $\sigma>0$ and $\beta_{1}(\sigma)<\beta<\beta_{2}(\sigma)$, there exists one and only one curve $\beta=\beta_{5}(\sigma)$ on the $(\sigma, \beta)$-plane on which $h_{\rho}^{-}=h_{2}$ or $h_{\rho}^{-}=h_{3}$. Moreover, the curves $\beta=\beta_{1}(\sigma), \beta=\beta_{2}(\sigma)$, $\beta=\beta_{4}(\sigma)$, and $\beta=\beta_{5}(\sigma)$ are tangent at the point $\left(\sigma_{1 s}, \beta_{1 s}\right)$. The curve $\beta=\beta_{3}(\sigma)$ intersects with the curves $\beta=\beta_{1}(\sigma)$, $\beta=\beta_{4}(\sigma)$, and $\beta=\beta_{5}(\sigma)$ at the points $\left(\sigma_{2 s}, \beta_{2 s}\right),\left(\sigma_{3 s}, \beta_{3 s}\right)$, and $\left(\sigma_{4 s}, \beta_{4 s}\right)\left(0<\sigma_{4 s}<\sigma_{3 s}<\sigma_{2 s}<\sigma_{1 s}\right)$, respectively.

(2) For $\sigma=0$, there exists a bifurcation point $A\left(0,-2 \epsilon / \varrho^{2}\right)$ on $(\sigma, \beta)$-plane on which $h_{\varrho}^{+}=h_{\varrho}^{-}=h_{2}$ when $\beta=-2 \epsilon / \varrho^{2}$, $h_{\varrho}^{+}=h_{\varrho}^{-}<h_{2}$ when $\beta<-2 \epsilon / \varrho^{2}$, and $h_{\varrho}^{+}=h_{\varrho}^{-}>h_{2}$ when $-2 \epsilon / Q^{2}<\beta<-\epsilon / Q^{2}$. 


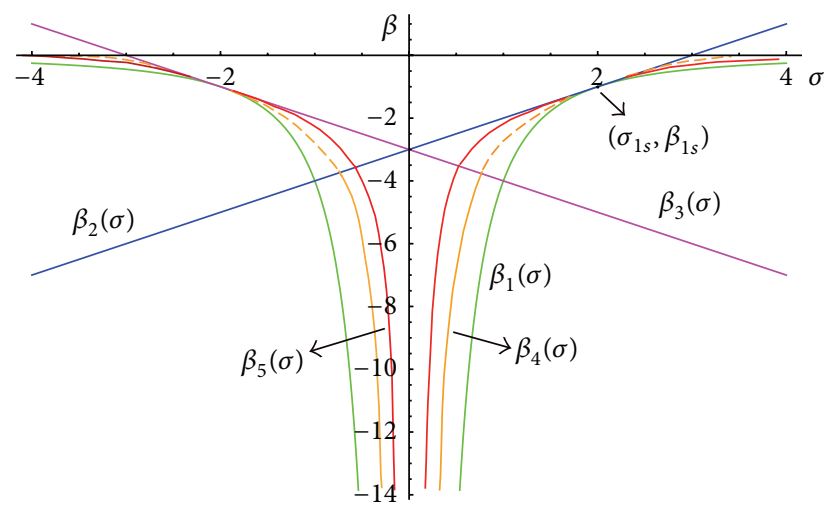

FIgURE 1: The bifurcation curves in the $(\sigma, \beta)$-parameter plane for $\epsilon>0$.

According to the above analysis and Lemma 1, we obtain the following proposition on the bifurcation curves of the phase portraits of system (15) for $\epsilon>0$.

Proposition 2. When $\epsilon>0$, for system (15), in $(\sigma, \beta)$ parameter plane, there exist five bifurcation curves (see Figure 1):

$$
\begin{aligned}
& \beta=\beta_{1}(\sigma)=-\frac{4 \epsilon^{3}}{27 \sigma^{2}}, \\
& \beta=\beta_{2}(\sigma)=\frac{\sigma-\epsilon \varrho}{\varrho^{3}}, \\
& \beta=\beta_{3}(\sigma)=\frac{-\sigma-\epsilon \varrho}{\varrho^{3}}, \\
& \beta=\beta_{4}(\sigma) \quad\left(|\sigma| \leq \sigma_{3 s}\right), \\
& \beta=\beta_{5}(\sigma) .
\end{aligned}
$$

These five curves divide the right-half $(\sigma, \beta)$-parameter plane into thirty-one regions as follows:

$$
\begin{aligned}
& A_{1}:\left\{(\sigma, \beta) \mid \sigma_{1 \mathrm{~s}}<\sigma<\epsilon \varrho, \beta=\beta_{2}(\sigma)\right\}, \\
& A_{2}:\left\{(\sigma, \beta) \mid \sigma>\sigma_{1 \mathrm{~s}}, \beta_{5}(\sigma)<\beta<\beta_{2}(\sigma)\right\}, \\
& A_{3}:\left\{(\sigma, \mathrm{b}) \mid \sigma>\sigma_{1 \mathrm{~s}}, \beta=\beta_{5}(\sigma)\right\}, \\
& A_{4}:\left\{(\sigma, \beta) \mid \sigma>\sigma_{1 \mathrm{~s}}, \beta_{1}(\sigma)<\beta<\beta_{5}(\sigma)\right\}, \\
& A_{5}:\left\{(\sigma, \beta) \mid \sigma>\sigma_{1 \mathrm{~s}}, \beta=\beta_{1}(\sigma)\right\}, \\
& A_{6}:\left\{(\sigma, \beta) \mid \sigma>\sigma_{2 \mathrm{~s}}, \beta_{3}(\sigma)<\beta<\beta_{1}(\sigma)\right\}, \\
& A_{7}:\left\{(\sigma, \beta) \mid \sigma<\sigma_{2 \mathrm{~s}}, \beta=\beta_{3}(\sigma)\right\}, \\
& A_{8}:\left\{(\sigma, \beta) \mid \sigma>0, \beta<\max \left\{\beta_{1}(\sigma), \beta_{3}(\sigma)\right\}\right\}, \\
& A_{9}:(\sigma, \beta)=\left(\sigma_{2 \mathrm{~s}}, \beta_{2 \mathrm{~s}}\right), \\
& A_{10}:\left\{(\sigma, \beta) \mid 0<\sigma<\sigma_{2 \mathrm{~s}}, \beta=\beta_{1}(\sigma)\right\}, \\
& A_{11}:\left\{(\sigma, \beta) \mid \sigma<\sigma_{2 \mathrm{~s}}, \beta_{1}(\sigma)<\beta<\min \left\{\beta_{3}(\sigma),\right.\right. \\
& \left.\left.\beta_{4}(\sigma)\right\}\right\}, \\
& A_{12}:\left\{(\sigma, \beta) \mid \sigma<\sigma_{3 \mathrm{~s}}, \beta=\beta_{4}(\sigma)\right\}, \\
& A_{13}:\left\{(\sigma, \beta) \mid \sigma>0, \beta_{4}(\sigma)<\beta<\beta_{5}(\sigma)\right\}, \\
& A_{14}:\left\{(\sigma, \beta) \mid \sigma<\sigma_{4 \mathrm{~s}}, \beta=\beta_{5}(\sigma)\right\},
\end{aligned}
$$

$$
\begin{aligned}
& A_{15}:\left\{(\sigma, \beta) \mid \sigma>0, \beta_{5}(\sigma)<\beta<\beta_{3}(\sigma)\right\}, \\
& A_{16}:\left\{(\sigma, \beta) \mid \sigma=0, \beta<-2 \epsilon / \varrho^{2}\right\}, \\
& A_{17}:(\sigma, \beta)=\left(0,-2 \epsilon / \varrho^{2}\right), \\
& A_{18}:\left\{(\sigma, \beta) \mid \sigma=0,-2 \epsilon / \varrho^{2}<\beta<-\epsilon / \varrho^{2}\right\}, \\
& A_{19}:(\sigma, \beta)=\left(0,-\epsilon / \varrho^{2}\right), \\
& A_{20}:\left\{(\sigma, \beta) \mid 0<\sigma<\sigma_{4 s}, \beta=\beta_{3}(\sigma)\right\}, \\
& A_{21}:(\sigma, \beta)=\left(\sigma_{4 s}, \beta_{4 s}\right), \\
& A_{22}:\left\{(\sigma, \beta) \mid \sigma_{4 s}<\sigma<\sigma_{2 s}, \beta=\beta_{3}(\sigma)\right\}, \\
& A_{23}:\left\{(\sigma, \beta) \mid \sigma_{4 s}<\sigma<\sigma_{1 s}, \max \left\{\beta_{1}(\sigma), \beta_{3}(\sigma)\right\}<\right. \\
& \left.\beta<\beta_{5}(\sigma)\right\}, \\
& A_{24}:\left\{(\sigma, \beta) \mid \sigma_{4}^{s}<\sigma<\sigma_{1 s}, \beta=\beta_{5}(\sigma)\right\}, \\
& A_{25}:\left\{(\sigma, \beta) \mid \sigma_{4 s}<\sigma<\sigma_{1 s}, \max \left\{\beta_{3}(\sigma), \beta_{5}(\sigma)\right\}<\right. \\
& \left.\beta<\beta_{2}(\sigma)\right\}, \\
& A_{26}:\left\{(\sigma, \beta) \mid 0<\sigma<\sigma_{1 s}, \beta=\beta_{2}(\mathrm{~d})\right\}, \\
& A_{27}:(\sigma, \beta)=\left(\sigma_{1 s}, \beta_{1 s}\right), \\
& A_{28}:\left\{(\sigma, \beta) \mid 0 \leq \sigma<\epsilon \varrho, \beta>\beta_{2}(\sigma)\right\}, \\
& A_{29}:\left\{(\sigma, \beta) \mid \sigma \in \mathbb{R}, \beta>\max \left\{0, \beta_{2}(\sigma)\right\}\right\}, \\
& A_{30}:\left\{(\sigma, \beta) \mid \sigma>\epsilon \varrho, \beta=\beta_{2}(\sigma)\right\}, \\
& A_{31}:\left\{(\sigma, \beta) \mid \sigma>\epsilon \varrho, 0<\beta<\beta_{2}(\sigma)\right\} .
\end{aligned}
$$

In this case, the phase portraits of system (15) can be shown in Figure 2.

2.2. Case II: $\epsilon<0$. In this case, we have the following.

Proposition 3. When $\epsilon<0$, for system (15), in $(\sigma, \beta)$ parameter plane, there exist four parametric bifurcation curves (see Figure 3):

$$
\begin{aligned}
& \beta=\beta_{1}(\sigma)=-\frac{4 \epsilon^{3}}{27 \sigma^{2}}, \\
& \beta=\beta_{2}(\sigma)=\frac{\sigma-\epsilon \varrho}{\varrho^{3}}, \\
& \beta=\beta_{3}(\sigma)=\frac{-\sigma-\epsilon \varrho}{\varrho^{3}}, \quad \sigma=0 .
\end{aligned}
$$

These four curves divide the right-half $(\sigma, \beta)$-parameter plane into twenty-two regions:

$$
\begin{aligned}
& B_{1}:\left\{(\sigma, \beta) \mid \sigma_{1 s}<\sigma<\epsilon \varrho, \beta=\beta_{3}(\sigma)\right\}, \\
& B_{2}:\left\{(\sigma, \beta) \mid \sigma>\sigma_{1 s}, \max \left\{0, \beta_{3}(\sigma)\right\}<\beta<\beta_{1}(\sigma)\right\}, \\
& B_{3}:\left\{(\sigma, \beta) \mid \sigma>\sigma_{1 s}, \beta=\beta_{1}(\sigma)\right\}, \\
& B_{4}:\left\{(\sigma, \beta) \mid \sigma>\sigma_{2 s}, \beta_{1}(\sigma)<\beta<\beta_{2}(\sigma)\right\}, \\
& B_{5}:(\sigma, \beta)=\left(\sigma_{2 s}, \beta_{2 s}\right), \\
& B_{6}:\left\{(\sigma, \beta) \mid \sigma>\sigma_{2 s}, \beta=\beta_{2}(\sigma)\right\}, \\
& B_{7}:\left\{(\sigma, \beta) \mid \sigma>0, \beta>\max \left\{\beta_{1}(\sigma), \beta_{2}(\sigma)\right\}\right\}, \\
& B_{8}:\left\{(\sigma, \beta) \mid 0<\sigma<\sigma_{2 s}, \beta=\beta_{1}(\sigma)\right\}, \\
& B_{9}:\left\{(\sigma, \beta) \mid 0<\sigma<\sigma_{2 s}, \beta_{2}(\sigma)<\beta<\beta_{1}(\sigma)\right\}, \\
& B_{10}:\left\{(\sigma, \beta) \mid \sigma=0, \beta>-\epsilon / \varrho^{2}\right\},
\end{aligned}
$$




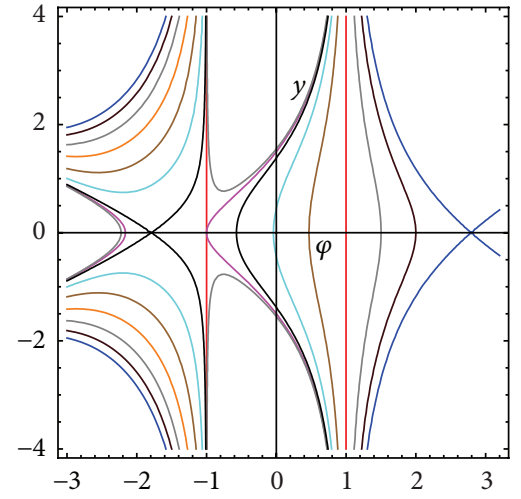

(1) $(\sigma, \beta) \in A_{1}$

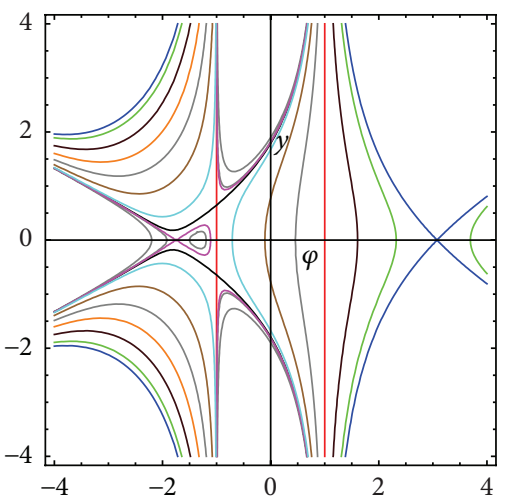

(4) $(\sigma, \beta) \in A_{4}$

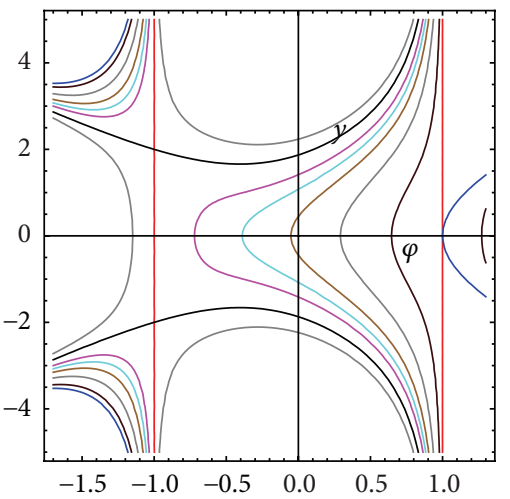

(7) $(\sigma, \beta) \in A_{7}$

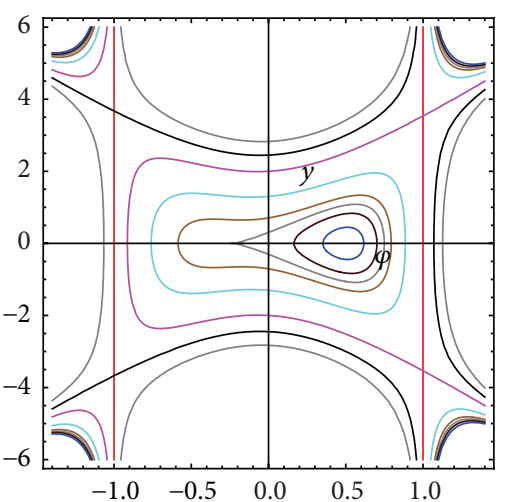

(10) $(\sigma, \beta) \in A_{10}$

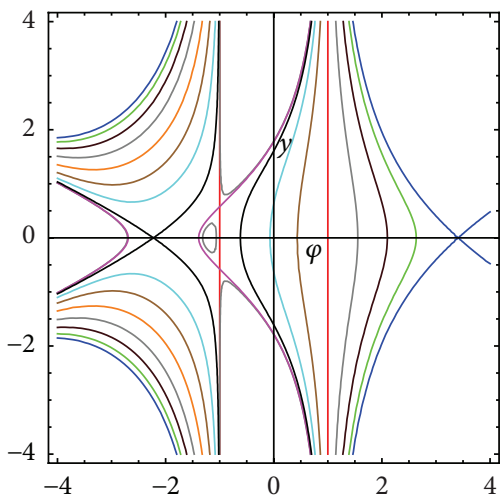

(2) $(\sigma, \beta) \in A_{2}$

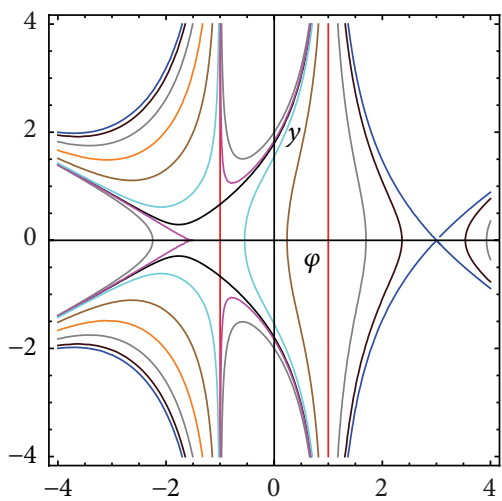

(5) $(\sigma, \beta) \in A_{5}$

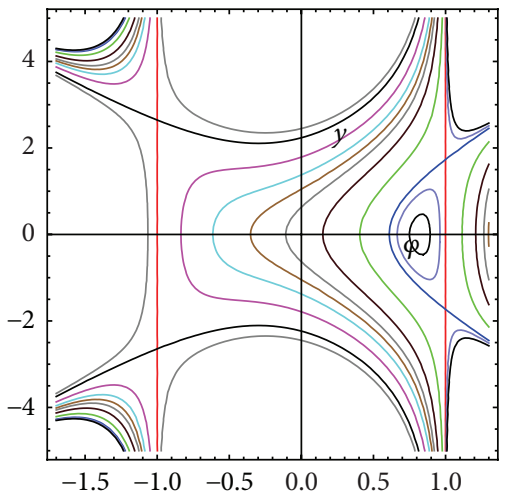

(8) $(\sigma, \beta) \in A_{8}$

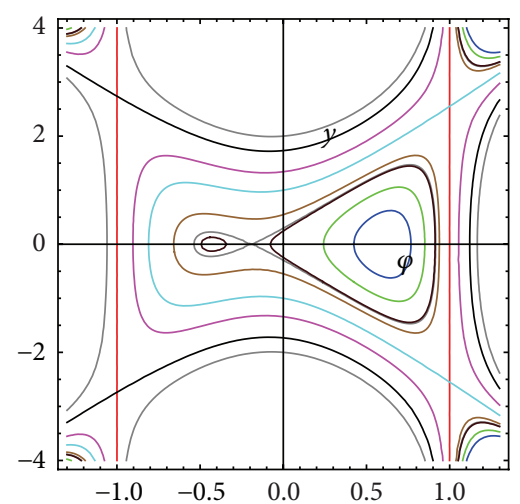

(11) $(\sigma, \beta) \in A_{11}$

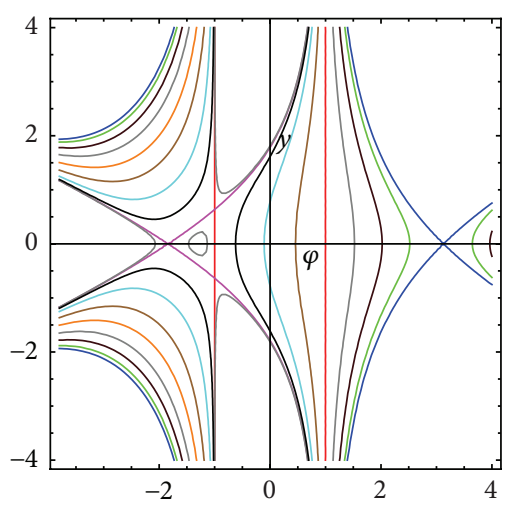

(3) $(\sigma, \beta) \in A_{3}$

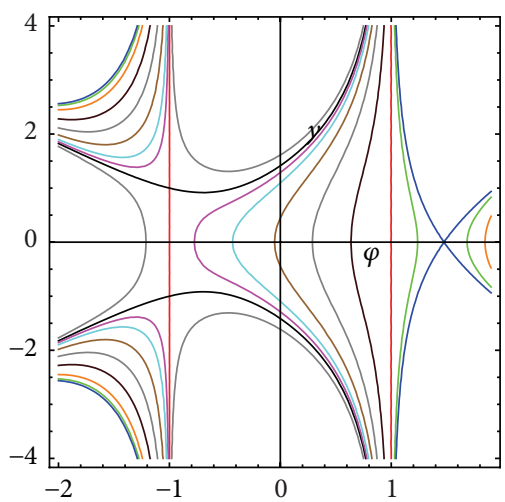

(6) $(\sigma, \beta) \in A_{6}$

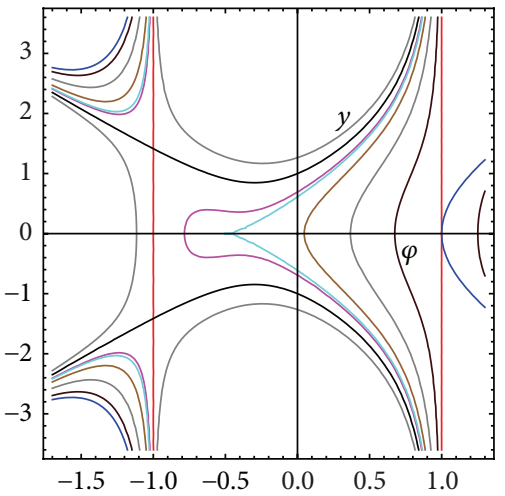

(9) $(\sigma, \beta) \in A_{9}$

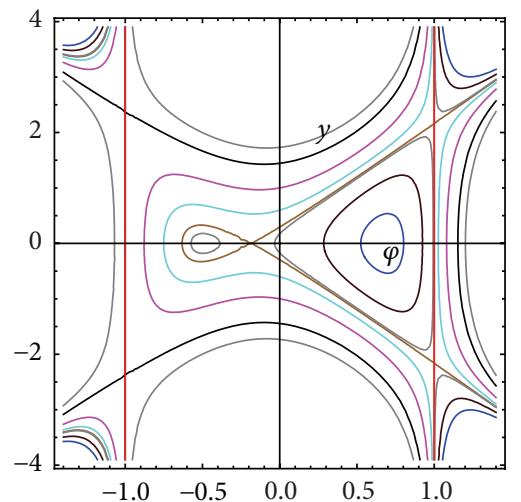

(12) $(\sigma, \beta) \in A_{12}$

Figure 2: Continued. 


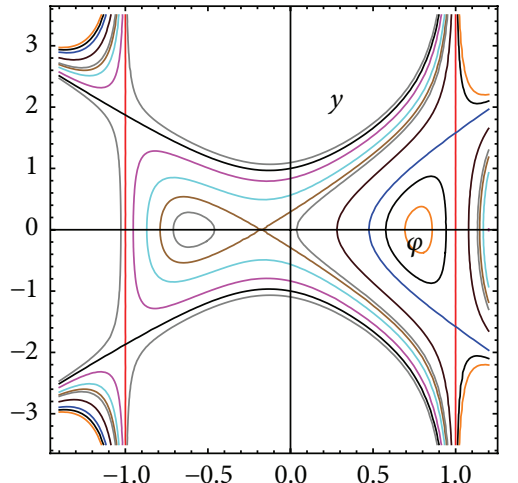

(13) $(\sigma, \beta) \in A_{13}$

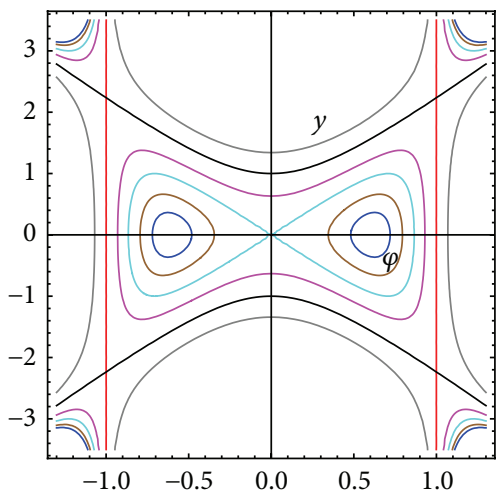

(16) $(\sigma, \beta) \in A_{16}$

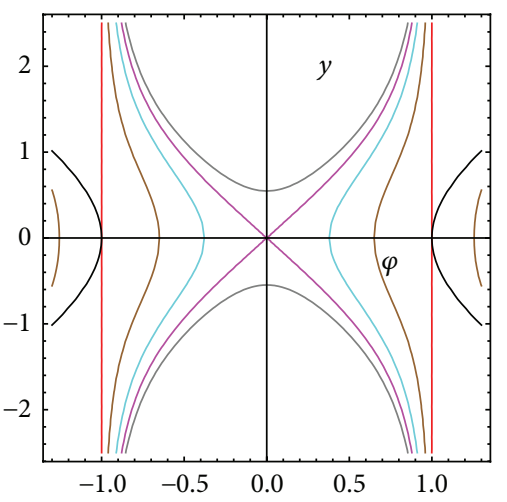

(19) $(\sigma, \beta) \in A_{19}$

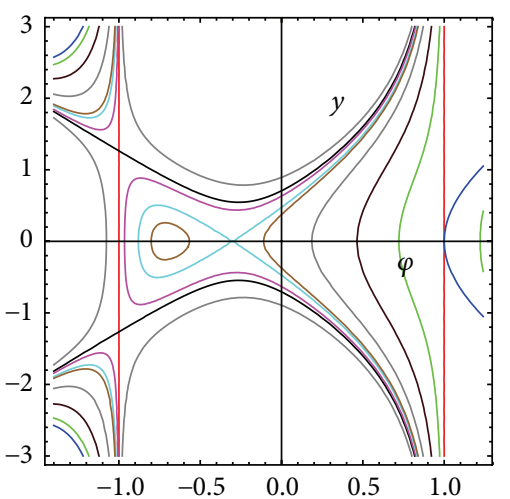

(22) $(\sigma, \beta) \in A_{22}$

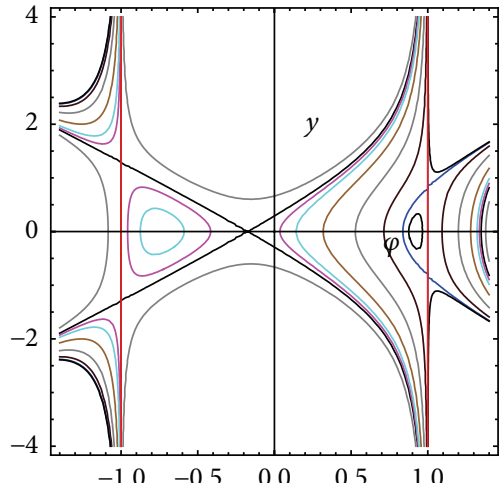

(14) $(\sigma, \beta) \in A_{14}$

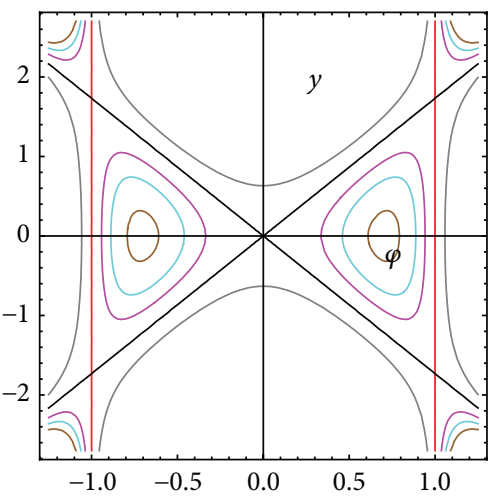

(17) $(\sigma, \beta) \in A_{17}$

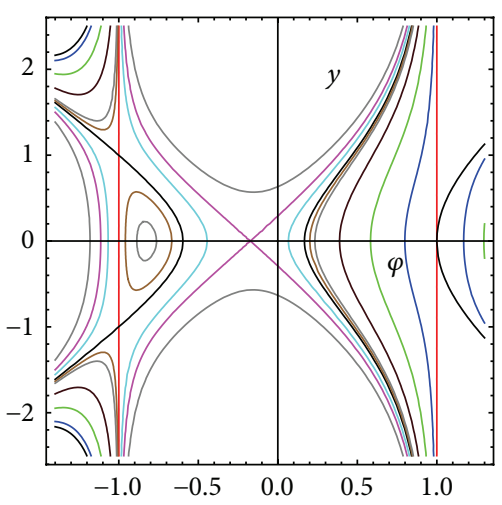

(20) $(\sigma, \beta) \in A_{20}$

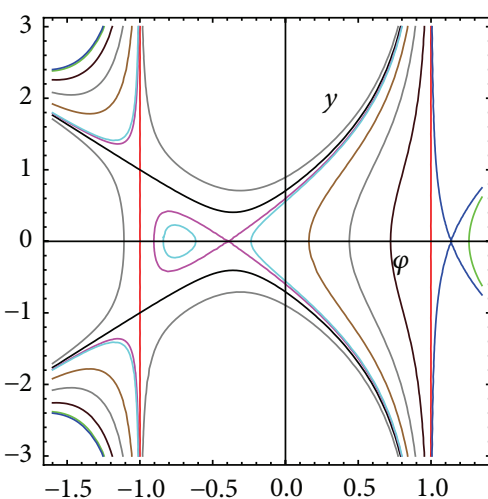

(23) $(\sigma, \beta) \in A_{23}$

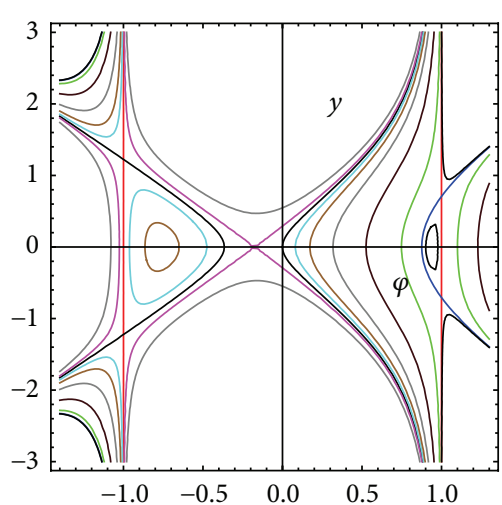

(15) $(\sigma, \beta) \in A_{15}$

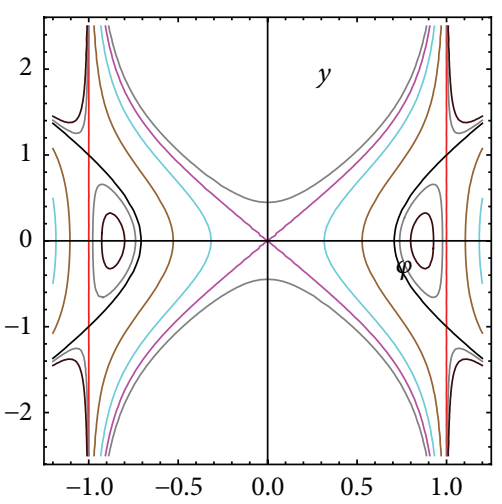

(18) $(\sigma, \beta) \in A_{18}$

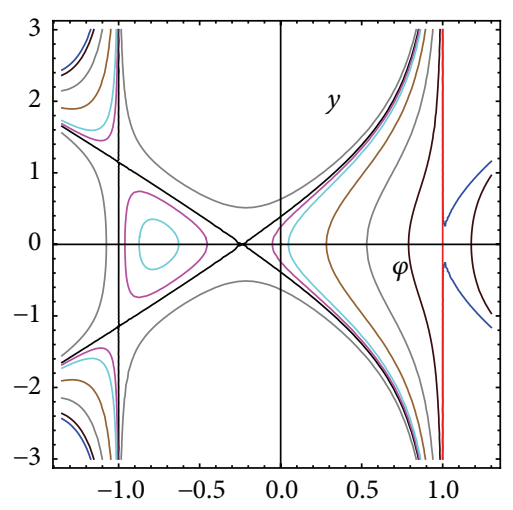

(21) $(\sigma, \beta) \in A_{21}$

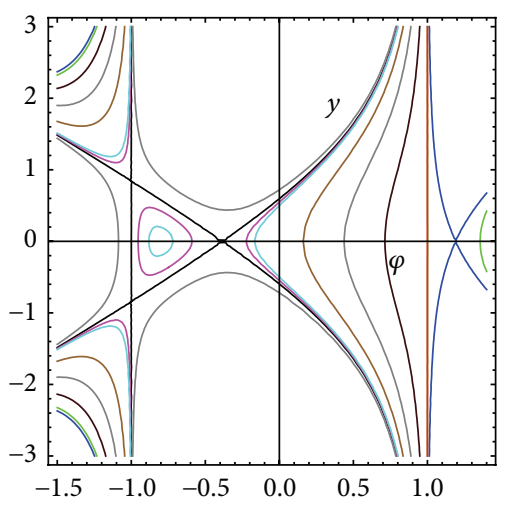

(24) $(\sigma, \beta) \in A_{24}$

FIGURE 2: Continued. 


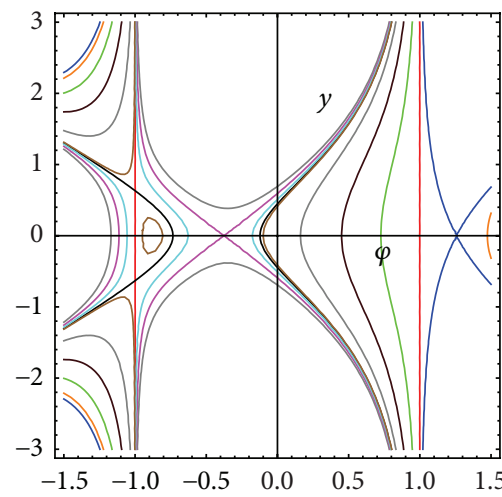

(25) $(\sigma, \beta) \in A_{25}$

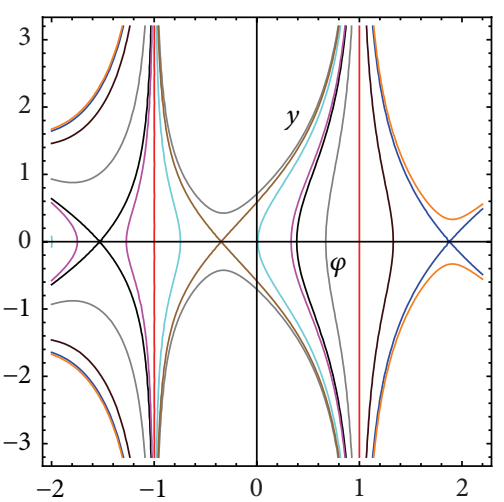

(28) $(\sigma, \beta) \in A_{28}$

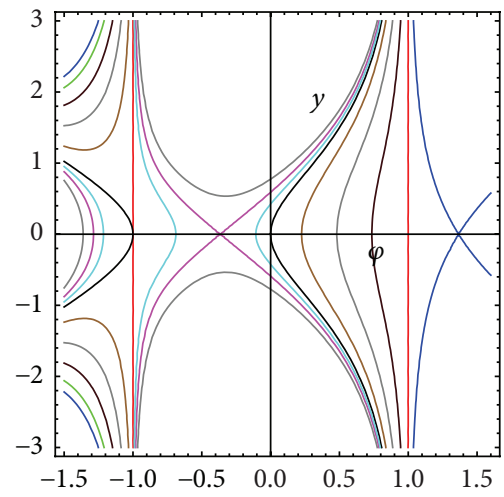

(26) $(\sigma, \beta) \in A_{26}$

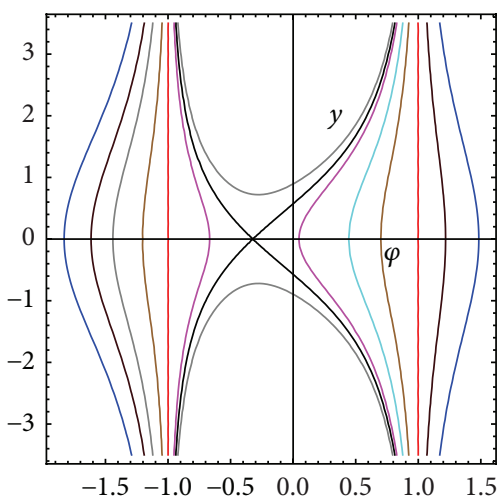

(29) $(\sigma, \beta) \in A_{29}$

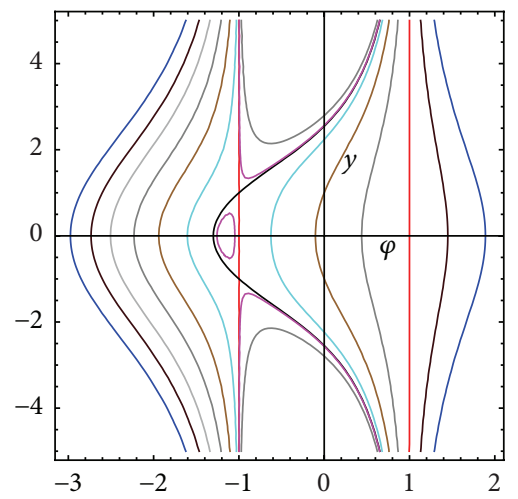

(31) $(\sigma, \beta) \in A_{31}$

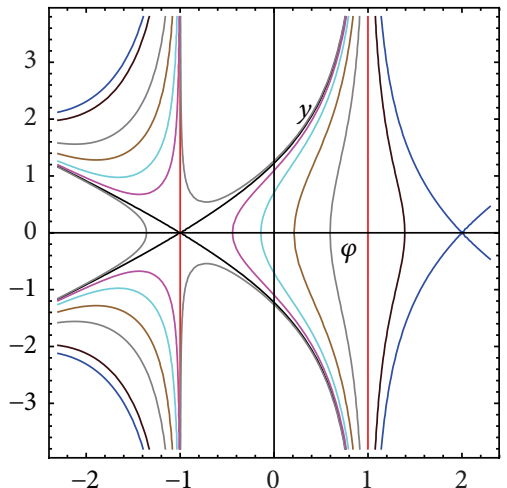

(27) $(\sigma, \beta) \in A_{27}$

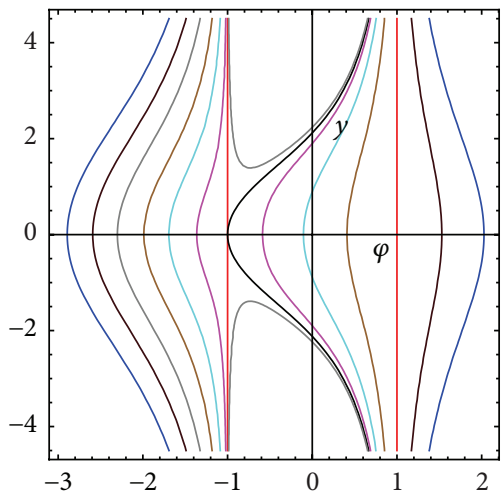

(30) $(\sigma, \beta) \in A_{30}$

FIGURE 2: The bifurcation of phase portraits of system (15) when $\epsilon>0$ and $\sigma \geq 0$.

$B_{11}:(\sigma, \beta)=\left(0,-\epsilon / \varrho^{2}\right)$,

$B_{12}:\left\{(\sigma, \beta) \mid 0<\sigma<\sigma_{2 s}, \beta=\beta_{2}(\sigma)\right\}$,

$B_{13}:\left\{(\sigma, \beta) \mid 0<\sigma<\sigma_{1 \mathrm{~s}}, \beta_{3}(\sigma)<\beta<\min \left\{\beta_{1}(\sigma)\right.\right.$, $\left.\left.\beta_{2}(\sigma)\right\}\right\}$,

$B_{14}:\left\{(\sigma, \beta) \mid \sigma_{4 \mathrm{~s}}<\sigma<\sigma_{1 \mathrm{~s}}, \beta=\beta_{1}(\sigma)\right\}$,

$B_{15}:(\sigma, \beta)=\left(\sigma_{1 \mathrm{~s}}, \beta_{1 \mathrm{~s}}\right)$,

$B_{16}:\left\{(\sigma, \beta) \mid 0<\sigma<\sigma_{1 s}, \beta=\beta_{3}(\sigma)\right\}$,

$B_{17}:\left\{(\sigma, \beta) \mid 0<\sigma<\epsilon \varrho, \beta<\beta_{3}(\sigma)\right\}$,
$B_{18}:\left\{(\sigma, \beta) \mid \sigma=0, \beta=-\epsilon / \varrho^{2}\right\}$,

$B_{19}:\{(\sigma, \beta) \mid \sigma=0, \beta<0\}$,

$B_{20}:\left\{(\sigma, \beta) \mid 0<\sigma<\epsilon \varrho, \beta<\min \left\{0, \beta_{3}(\sigma)\right\}\right\}$,

$B_{21}:\left\{(\sigma, \beta) \mid \sigma>\epsilon \varrho, \beta=\beta_{3}(\sigma)\right\}$,

$B_{22}:\left\{(\sigma, \beta) \mid \sigma>\epsilon \varrho, \beta_{3}(\sigma)<\beta<0\right\}$.

Based on Proposition 3, we obtain the phase portraits of system (15) which are shown in Figure 4. 


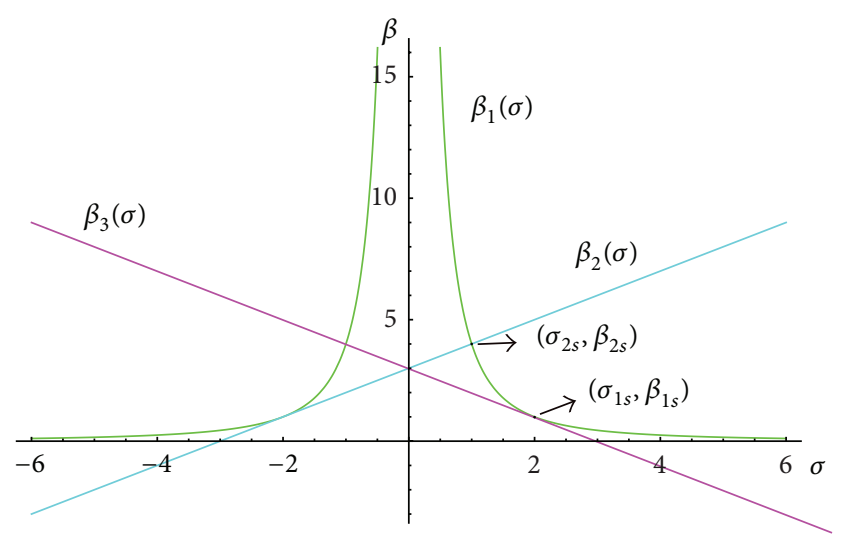

FIgURE 3: The bifurcation curves in the $(\sigma, \beta)$-parameter plane for $\epsilon<0$.

\section{Single Peak Soliton Solutions}

In this section, we study classification of single peak soliton solutions of (10) by using the phase portraits given in Section 2 . Let $C^{k}(\Omega)$ denote the set of all $k$ times continuously differentiable functions on the open set $\Omega . L_{\text {loc }}^{p}(\mathbb{R})$ refer to the set of all functions whose restriction on any compact subset is $L^{p}$ integrable. $H_{\text {loc }}^{1}(\mathbb{R})$ stands for $H_{\text {loc }}^{1}(\mathbb{R})=\left\{\varphi \in L_{\text {loc }}^{2}(\mathbb{R}) \mid\right.$ $\left.\varphi^{\prime} \in L_{\text {loc }}^{2}(\mathbb{R})\right\}$.

To study single peak soliton solutions, we impose the boundary condition

$$
\lim _{\xi \rightarrow \pm \infty} \varphi=A
$$

where $A$ is a constant. In fact, the constant $A$ is equal to the horizontal coordinate of saddle point $E\left(\varphi_{e}, 0\right)$. Substituting the boundary condition (22) into (14) generates the following constant:

$$
h=A\left(\sigma+\frac{A \epsilon}{2}+\frac{A^{3} \beta}{4}\right) .
$$

So the ODE (14) becomes

$$
\left(\varphi^{\prime}\right)^{2}=\frac{(\varphi-A)^{2}\left(\beta \varphi^{2}+2 A \beta \varphi+3 A^{2} \beta+2 \epsilon\right)}{2\left(\varrho^{2}-\varphi^{2}\right)} .
$$

If $\beta\left(A^{2} \beta+\epsilon\right) \leq 0$, then (24) reduces to

$$
\left(\varphi^{\prime}\right)^{2}=\frac{\beta(\varphi-A)^{2}\left(\varphi-B_{1}\right)\left(\varphi-B_{2}\right)}{2\left(\varrho^{2}-\varphi^{2}\right)},
$$

where

$$
\begin{aligned}
& B_{1}=-A-\frac{\sqrt{-2 \beta\left(A^{2} \beta+\epsilon\right)}}{\beta}, \\
& B_{2}=-A+\frac{\sqrt{-2 \beta\left(A^{2} \beta+\epsilon\right)}}{\beta} .
\end{aligned}
$$
$\beta>0$.

From (26) we know that $B_{1}>B_{2}$ if $\beta<0$ and $B_{1}<B_{2}$ if

Definition 4. A function $\varphi(\xi)$ is said to be a single peak soliton solution for the $C(3,2,2)$ equation $(10)$ if $\varphi(\xi)$ satisfies the following conditions:

$\left(\mathrm{C}_{1}\right) \varphi(\xi)$ is continuous on $\mathbb{R}$ and has a unique peak point $\xi_{0}$, where $\varphi(\xi)$ attains its global maximum or minimum value.

$\left(\mathrm{C}_{2}\right) \varphi(\xi) \in C^{3}\left(\mathbb{R}-\left\{\xi_{0}\right\}\right)$ satisfies $(24)$ on $\mathbb{R}-\left\{\xi_{0}\right\}$.

$\left(\mathrm{C}_{3}\right) \varphi(\xi)$ satisfies the boundary condition (22).

Definition 5. A wave function $\varphi$ is called smooth soliton solution if $\varphi$ is smooth locally on either side of $\xi_{0}$ and $\lim _{\xi \uparrow \xi_{0}} \varphi^{\prime}(\xi)=\lim _{\xi \downarrow \xi_{0}} \varphi^{\prime}(\xi)=0$.

Definition 6. A wave function $\varphi$ is called peakon if $\varphi$ is smooth locally on either side of $\xi_{0}$ and $\lim _{\xi \uparrow \xi_{0}} \varphi^{\prime}(\xi)=$ $-\lim _{\xi \downarrow \xi_{0}} \varphi^{\prime}(\xi)=a, a \neq 0, a \neq \pm \infty$.

Definition 7. A wave function $\varphi$ is called cuspon if $\varphi$ is smooth locally on either side of $\xi_{0}$ and $\lim _{\xi \uparrow \xi_{0}} \varphi^{\prime}(\xi)=-\lim _{\xi \downarrow \xi_{0}} \varphi^{\prime}(\xi)=$ $\pm \infty$.

Without any loss of generality, we choose the peak point $\xi_{0}$ as vanishing, $\xi_{0}=0$.

Theorem 8. Assume that $u(x, t)=\varphi(\xi)=\varphi(x-c t)$ is a single peak soliton solution of the $C(3,2,2)$ equation (10) at the peak point $\xi_{0}=0$. Then, we have the following:

(i) If $\beta\left(A^{2} \beta+\epsilon\right)>0$, then $\varphi(0)=\varrho$ or $\varphi(0)=-\varrho$.

(ii) If $\beta\left(A^{2} \beta+\epsilon\right) \leq 0$, then $\varphi(0)=\varrho$ or $\varphi(0)=-\varrho$ or $\varphi(0)=B_{1}$ or $\varphi(0)=B_{2}$.

Proof. If $\varphi(0) \neq \pm \varrho$, then $\varphi(\xi) \neq \pm \varrho$ for any $\xi \in \mathbb{R}$ since $\varphi(\xi) \in C^{3}(\mathbb{R}-\{0\})$. Differentiating both sides of (24) yields $\varphi \in C^{\infty}(\mathbb{R})$.

(i) When $\beta\left(A^{2} \beta+\epsilon\right)>0$, if $\varphi(0) \neq \varrho$ and $\varphi(0) \neq-\varrho$, then $\varphi \in C^{\infty}(\mathbb{R})$. By the definition of single peak soliton we have $\varphi^{\prime}(0)=0$. However, by $(24)$ we must have $\varphi(0)=A$, which contradicts the fact that 0 is the unique peak point.

(ii) When $\beta\left(A^{2} \beta+\epsilon\right) \leq 0$, if $\varphi(0) \neq \varrho$ and $\varphi(0) \neq-\varrho$, by (24) we know $\varphi^{\prime}(0)$ exists and $\varphi^{\prime}(0)=0$ since 0 is a peak point. Thus, we obtain $\varphi(0)=B_{1}$ or $\varphi(0)=B_{2}$ from (25), since $\varphi(0)=A$ contradicts the fact that 0 is the unique peak point.

Now we give the following theorem on the classification of single peak solitons of (10). The idea is inspired by the study of the traveling waves of Camassa-Holm equation $[18,19]$.

Theorem 9. Assume that $u(x, t)=\varphi(x-c t)$ is a single peak soliton solution of the $C(3,2,2)$ equation (10) at the peak point $\xi_{0}=0$. Then, we have the following solution classification:

(i) If $|\varphi(0)| \neq \varrho$, then $\varphi(\xi) \in C^{\infty}(\mathbb{R})$, and $\varphi$ is a smooth soliton solution. 


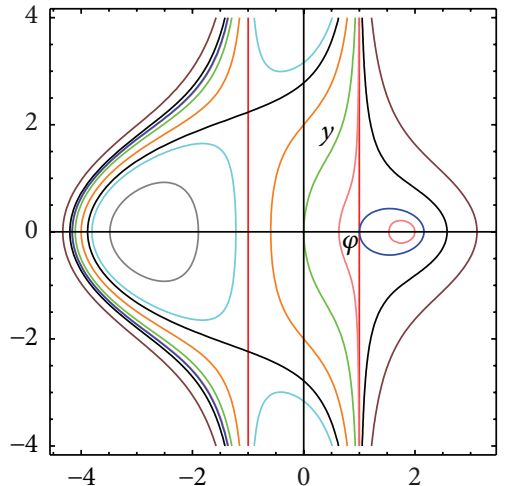

(1) $(\sigma, \beta) \in B_{1}$

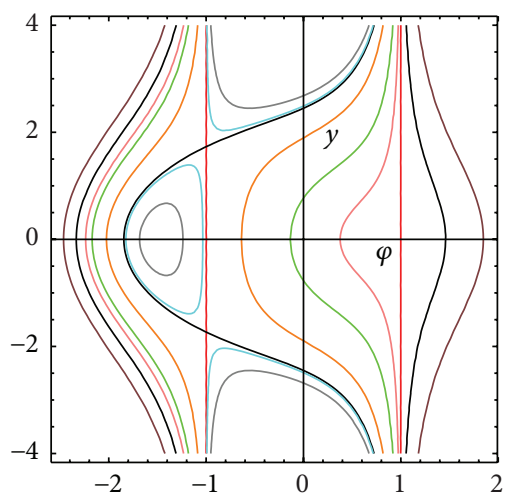

(4) $(\sigma, \beta) \in B_{4}$

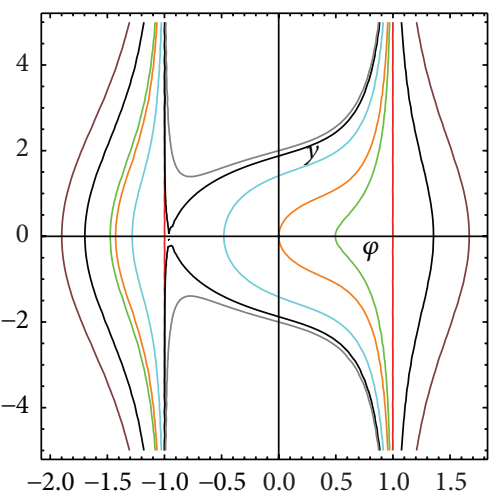

(7) $(\sigma, \beta) \in B_{7}$

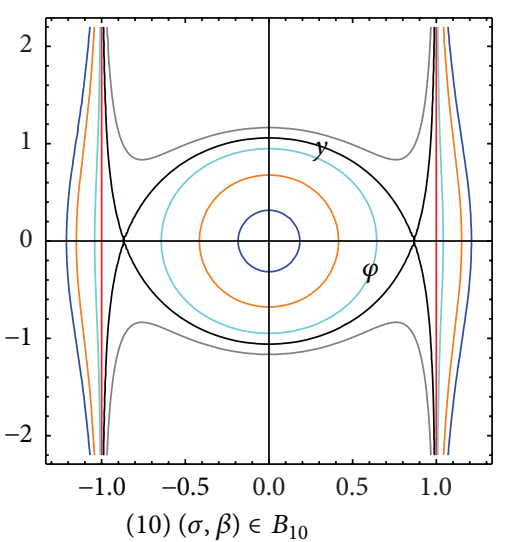

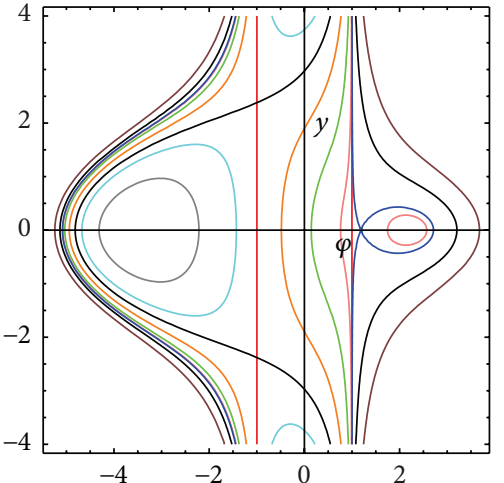

(2) $(\sigma, \beta) \in B_{2}$

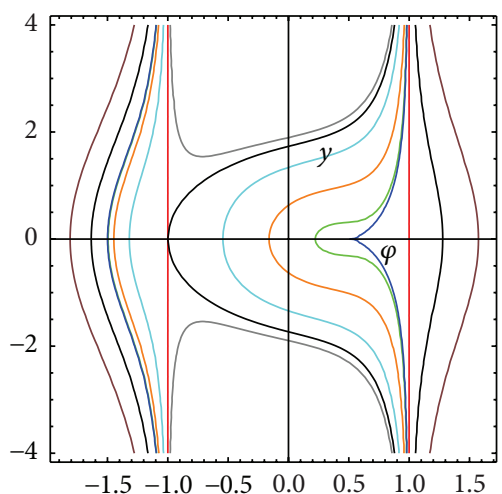

(5) $(\sigma, \beta) \in B_{5}$

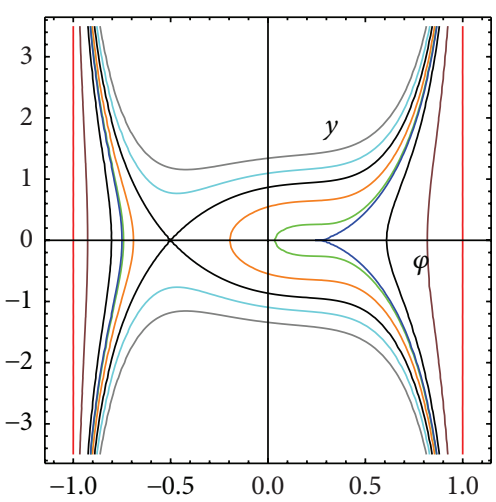

(8) $(\sigma, \beta) \in B_{8}$

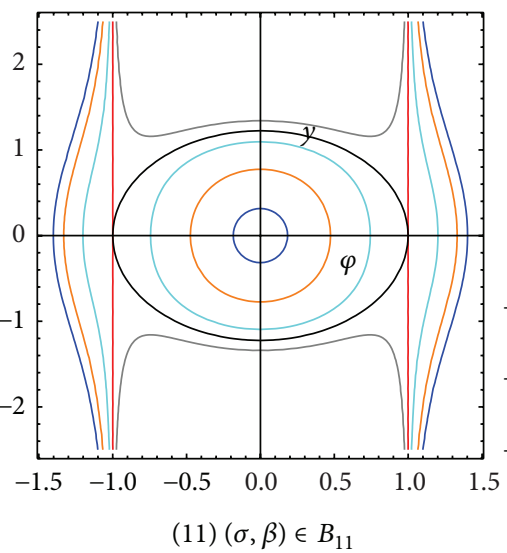

FIgUre 4: Continued.

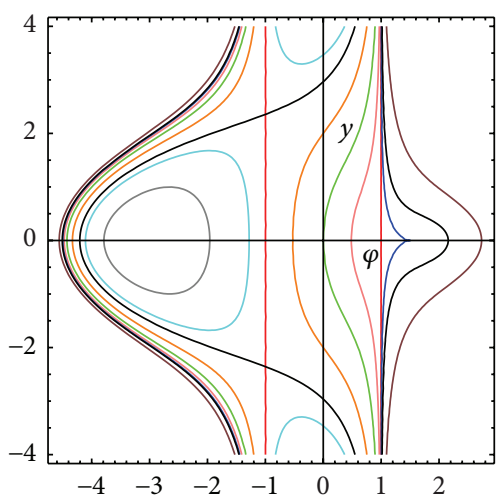

(3) $(\sigma, \beta) \in B_{3}$

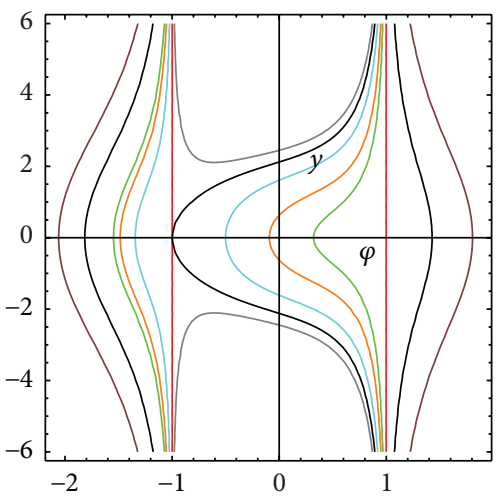

(6) $(\sigma, \beta) \in B_{6}$

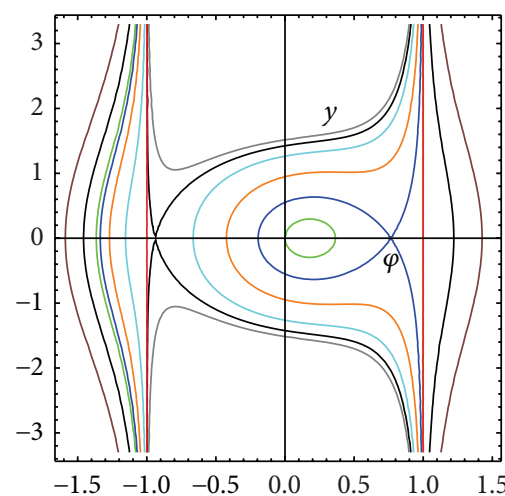

(9) $(\sigma, \beta) \in B_{9}$

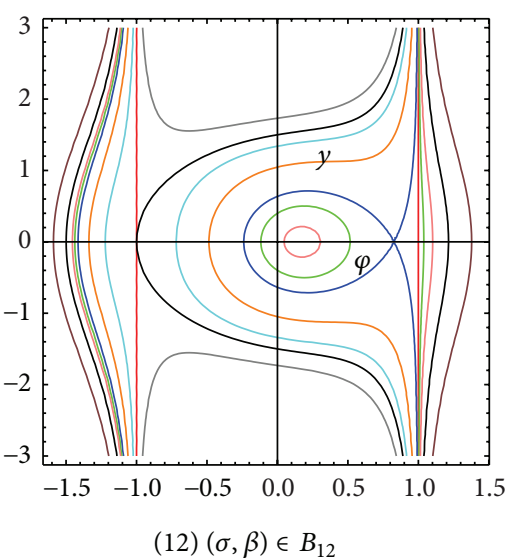

(12) $(\sigma, \beta) \in B_{12}$ 


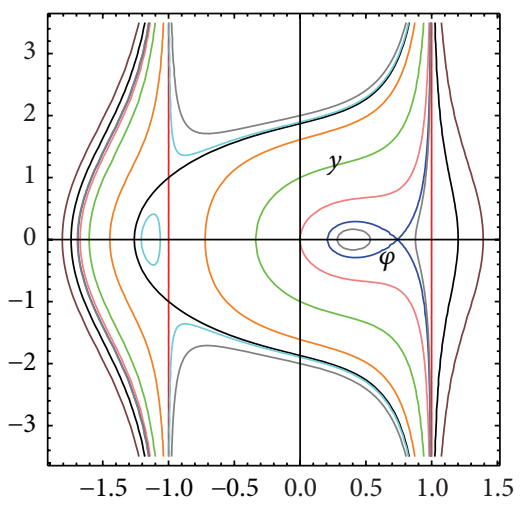

(13) $(\sigma, \beta) \in B_{13}$

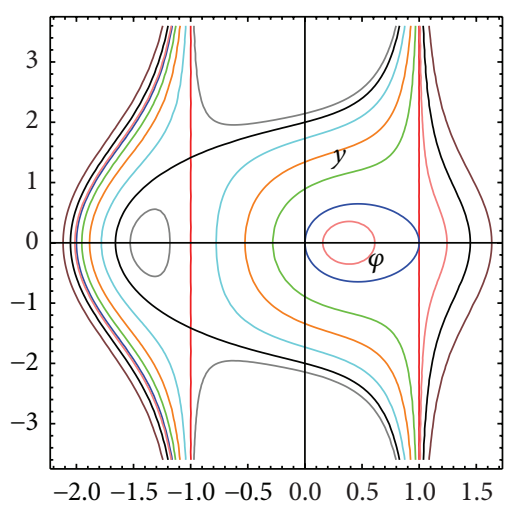

(16) $(\sigma, \beta) \in B_{16}$

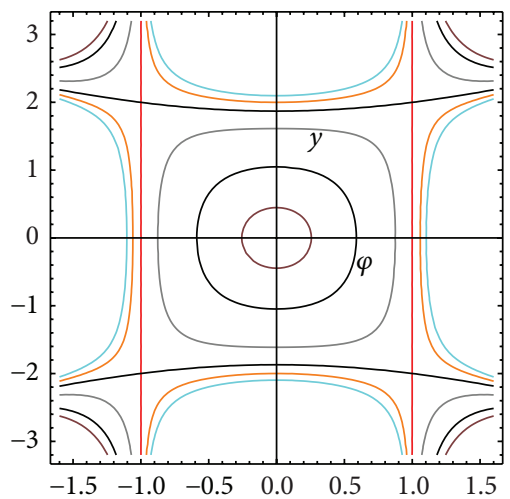

(19) $(\sigma, \beta) \in B_{19}$

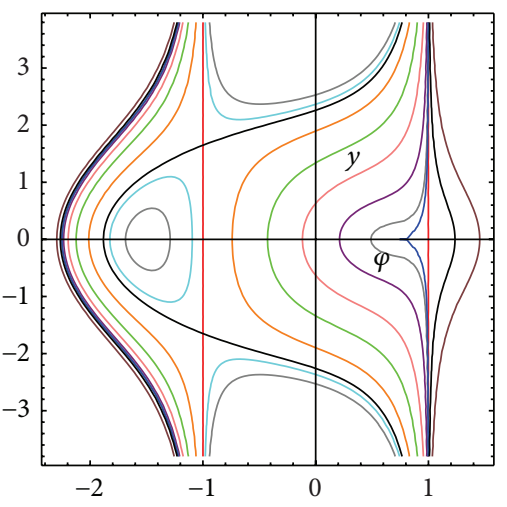

(14) $(\sigma, \beta) \in B_{14}$

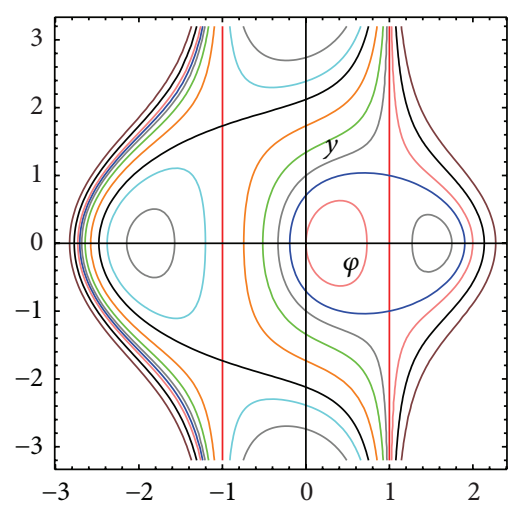

(17) $(\sigma, \beta) \in B_{17}$
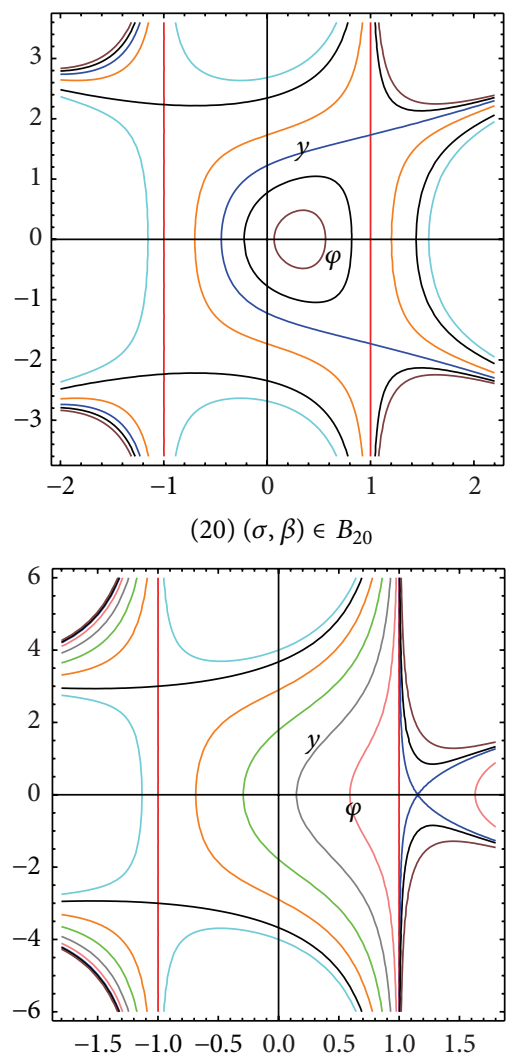

(22) $(\sigma, \beta) \in B_{22}$

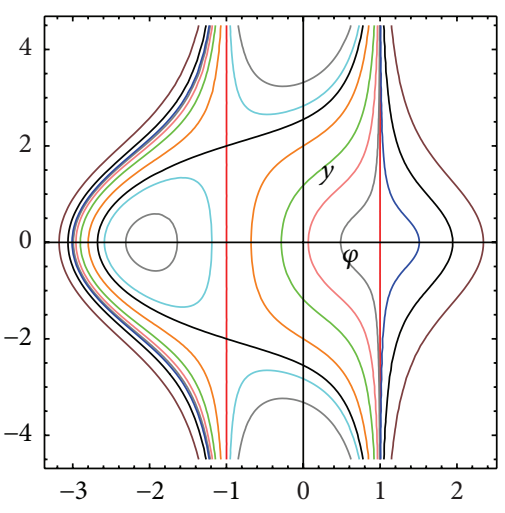

(15) $(\sigma, \beta) \in B_{15}$

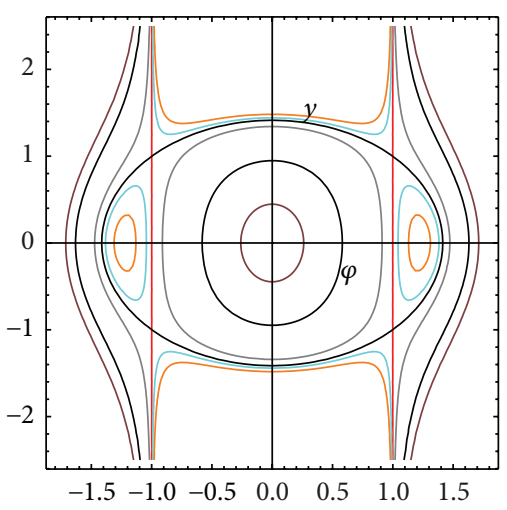

(18) $(\sigma, \beta) \in B_{18}$

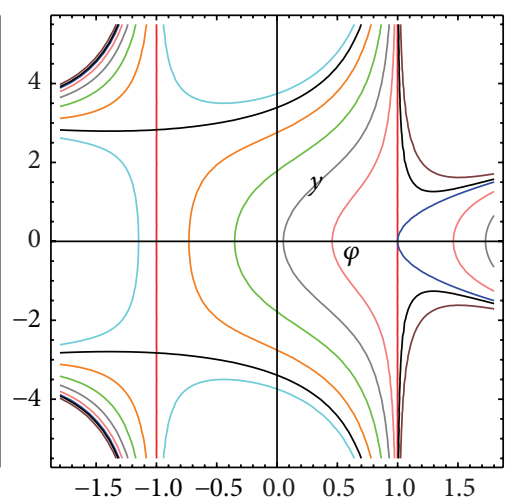

(21) $(\sigma, \beta) \in B_{21}$

Figure 4: The bifurcation of phase portraits of system (15) when $\epsilon<0$ and $\sigma \geq 0$. 
(ii) If $\varphi(0)=\varrho \neq B_{1}$, then $\varphi$ is a cuspon solution and $\varphi$ has the following asymptotic behavior:

$$
\begin{aligned}
\varphi(\xi)-\varrho & =\lambda_{1}|\xi|^{2 / 3}+O\left(|\xi|^{4 / 3}\right), \quad \xi \longrightarrow 0, \\
\varphi^{\prime}(\xi) & =\frac{2}{3} \lambda_{1}|\xi|^{-1 / 3} \operatorname{sgn}(\xi)+O\left(|\xi|^{1 / 3}\right),
\end{aligned}
$$

$$
\xi \longrightarrow 0
$$

where $\lambda_{1}= \pm\left(9\left|\beta \varrho^{2}+2 A \beta \varrho+3 A^{2} \beta+2 \epsilon\right|(\varrho-A)^{2} / 16 \varrho\right)^{1 / 3}$. Thus, $\varphi(\xi) \notin H_{\text {loc }}^{1}(\mathbb{R})$.

(iii) If $\varphi(0) \stackrel{=}{=}-\varrho \neq B_{2}$, then $\varphi$ is a cuspon solution and $\varphi$ has the following asymptotic behavior:

$$
\begin{aligned}
\varphi(\xi)+\varrho & =\lambda_{2}|\xi|^{2 / 3}+O\left(|\xi|^{4 / 3}\right), \quad \xi \longrightarrow 0, \\
\varphi^{\prime}(\xi) & =\frac{2}{3} \lambda_{2}|\xi|^{-1 / 3} \operatorname{sgn}(\xi)+O\left(|\xi|^{1 / 3}\right),
\end{aligned}
$$

\section{$\xi \longrightarrow 0$,}

where $\lambda_{2}= \pm\left(9\left|\beta \varrho^{2}-2 A \beta \varrho+3 A^{2} \beta+2 \epsilon\right|(\varrho+A)^{2} / 16 \varrho\right)^{1 / 3}$. Thus, $\varphi(\xi) \notin H_{\text {loc }}^{1}(\mathbb{R})$.

(iv) If $\varphi(0)=\varrho=B_{1}$ and $A \neq 0$, then $\varphi$ is a peakon-like solution and

$$
\begin{aligned}
\varphi(\xi)-\varrho & =\lambda_{3}|\xi|+O\left(|\xi|^{2}\right), \quad \xi \longrightarrow 0, \\
\varphi^{\prime}(\xi) & =\lambda_{3} \operatorname{sgn}(\xi)+O(|\xi|), \quad \xi \longrightarrow 0,
\end{aligned}
$$

where $\lambda_{3}= \pm|A-\varrho| \sqrt{\beta\left(B_{2}-\varrho\right) / 4 \varrho}$.

(v) If $\varphi(0)=-\varrho=B_{2}$ and $A \neq 0$, then $\varphi$ is a peakon-like solution and

$$
\begin{aligned}
\varphi(\xi)+\varrho & =\lambda_{4}|\xi|+O\left(|\xi|^{2}\right), \quad \xi \longrightarrow 0, \\
\varphi^{\prime}(\xi) & =\lambda_{4} \operatorname{sgn}(\xi)+O(|\xi|), \quad \xi \longrightarrow 0,
\end{aligned}
$$

where $\lambda_{4}= \pm|A+\varrho| \sqrt{-\beta\left(B_{1}+\varrho\right) / 4 \varrho}$.

(vi) If $\varphi(0)=\varrho=B_{1}$ and $A=0$, then $\varphi$ gives the peakon solution $\varrho \exp (-\sqrt{-\beta / 2}|x-c t|)$.

(vii) If $\varphi(0)=-\varrho=B_{2}$ and $A=0$, then $\varphi$ gives the peakon solution $-\varrho \exp (-\sqrt{-\beta / 2}|x-c t|)$.

Proof. (vi) and (vii) are obvious. Let us prove (i), (ii), and (iv) in order.

(i) From the process of proofing of Theorem 8, we know that if $|\varphi(0)| \neq \varrho$, then $\varphi \in C^{\infty}(\mathbb{R})$ and $\varphi$ is a smooth soliton solution.

(ii) If $\varphi(0)=\varrho \neq B_{1}$, then by the definition of single peak soliton we have $A \neq \varrho$; thus, $\beta \varphi^{2}+2 A \beta \varphi+3 A^{2} \beta+2 \epsilon$ does not contain the factor $\varphi-\varrho$. From (24), we obtain

$$
\begin{aligned}
\varphi^{\prime}= & \operatorname{sgn}(A-\varrho) \frac{|\varphi-A|}{\sqrt{2\left|\varphi^{2}-\varrho^{2}\right|}} \\
& \cdot \sqrt{\left|\beta \varphi^{2}+2 A \beta \varphi+3 A^{2} \beta+2 \epsilon\right|} \operatorname{sgn}(\xi) .
\end{aligned}
$$

Let $l_{1}(\varphi)=\sqrt{2(\varphi+\varrho)} /|\varphi-A| \sqrt{\left|\beta \varphi^{2}+2 A \beta \varphi+3 A^{2} \beta+2 \epsilon\right|}$; then, $l_{1}(\varrho)=2 \sqrt{\varrho} /|\varrho-A| \sqrt{\left|\beta \varrho^{2}+2 A \beta \varrho+3 A^{2} \beta+2 \epsilon\right|}$, and

$$
\int l_{1}(\varphi) \sqrt{|\varphi-\varrho|} d \varphi=\int \operatorname{sgn}(A-\varrho) \operatorname{sgn}(\xi) d \xi .
$$

Inserting $l_{1}(\varphi)=l_{1}(\varrho)+O(|\varphi-\varrho|)$ into (32) and using the initial condition $\varphi(0)=\varrho$, we obtain

$$
\frac{2 l_{1}(\varrho)}{3}|\varphi-\varrho|^{3 / 2}(1+O(|\varphi-\varrho|))=|\xi| ;
$$

thus,

$$
\begin{aligned}
\varphi-\varrho & = \pm\left(\frac{3}{2 l_{1}(\varrho)}\right)^{2 / 3}|\xi|^{2 / 3}(1+O(|\varphi-\varrho|))^{-2 / 3} \\
& = \pm\left(\frac{3}{2 l_{1}(\varrho)}\right)^{2 / 3}|\xi|^{2 / 3}(1+O(|\varphi-\varrho|)),
\end{aligned}
$$

which implies $\varphi-\varrho=O\left(|\xi|^{2 / 3}\right)$. Therefore, we have

$$
\begin{aligned}
\varphi(\xi) & =\varrho \pm\left(\frac{3}{2 l_{1}(\varrho)}\right)^{2 / 3}|\xi|^{2 / 3}+O\left(|\xi|^{4 / 3}\right) \\
& =\varrho+\lambda_{1}|\xi|^{2 / 3}+O\left(|\xi|^{4 / 3}\right), \quad \xi \longrightarrow 0, \\
\lambda_{1}= \pm\left(\frac{3}{2 l_{1}(\varrho)}\right)^{2 / 3} & \\
= & \pm\left(\frac{9\left|\beta \varrho^{2}+2 A \beta \varrho+3 A^{2} \beta+2 \epsilon\right|(\varrho-A)^{2}}{16 \varrho}\right)^{1 / 3}, \\
\varphi^{\prime}(\xi) & =\frac{2}{3} \lambda_{1}|\xi|^{-1 / 3} \operatorname{sgn}(\xi)+O\left(|\xi|^{1 / 3}\right), \quad \xi \longrightarrow 0 .
\end{aligned}
$$

So, $\varphi(\xi) \notin H_{\text {loc }}^{1}(\mathbb{R})$.

(iii) Similar to the proof of (ii), we ignore it in this paper. (iv) If $\varphi(0)=\varrho=B_{1}$ and $A \neq 0$, then from (25) we obtain

$$
\varphi^{\prime}=\sqrt{-\frac{\beta}{2}} \operatorname{sgn}(A-\varrho)|\varphi-A| \sqrt{\frac{\varphi-B_{2}}{\varphi+\varrho}} \operatorname{sgn}(\xi) .
$$

Let $l_{2}(\varphi)=(1 /|\varphi-A|) \sqrt{(\varphi+\varrho) /\left(\varphi-B_{2}\right)}$; then, $l_{2}(\varrho)=(1 / \mid \varrho-$ $A \mid) \sqrt{2 \varrho /\left(\varrho-B_{2}\right)}$ and

$$
\int l_{2}(\varphi) d \varphi=\sqrt{-\frac{\beta}{2}} \int \operatorname{sgn}(A-\varrho) \operatorname{sgn}(\xi) d \xi .
$$

Inserting $l_{2}(\varphi)=l_{2}(\varrho)+O(|\varphi-\varrho|)$ into (37) and using the initial condition $\varphi(0)=\varrho$, we obtain

$$
l_{2}(\varrho)(\varphi-\varrho)(1+O(|\varphi-\varrho|))^{-1}=\operatorname{sgn}(A-\varrho)|\xi| .
$$

Since

$$
\begin{aligned}
\operatorname{sgn}(\varphi-\varrho) \operatorname{sgn}(A-\varrho) & \geq 0, \\
\frac{1}{1+O(\varphi-\varrho)} & =1+O(\varphi-\varrho),
\end{aligned}
$$


we get

$$
|\varphi-\varrho|=\sqrt{-\frac{\beta}{2}} \frac{1}{l_{2}(\varrho)}|\xi|(1+O(\varphi-\varrho))
$$

which implies $|\varphi-\varrho|=O(|\xi|)$. Therefore, we have

$$
\begin{aligned}
\varphi(\xi) & =\varrho+\lambda_{3}|\xi|+O\left(|\xi|^{2}\right), \quad \xi \longrightarrow 0, \\
\varphi^{\prime}(\xi) & =\lambda_{3} \operatorname{sgn}(\xi)+O(|\xi|), \quad \xi \longrightarrow 0,
\end{aligned}
$$

where $\lambda_{3}= \pm|A-\varrho| \sqrt{\beta\left(B_{2}-\varrho\right) / 4 \varrho}$.

(v) Similar to the proof of (iv), we ignore it in this paper.

By virtue of Theorem 9, any single peak soliton for the $C(3,2,2)$ equation (10) must satisfy the following initial and boundary values problem:

$$
\begin{aligned}
& \left(\varphi^{\prime}\right)^{2}=\frac{\beta(\varphi-A)^{2}\left(\varphi-B_{1}\right)\left(\varphi-B_{2}\right)}{2\left(\varrho^{2}-\varphi^{2}\right)}:=L(\varphi), \\
& \varphi(0) \in\left\{\varrho,-\varrho, B_{1}, B_{2}\right\},
\end{aligned}
$$

$\lim _{|\xi| \rightarrow \infty} \varphi(\xi)=A$.

$L(\varphi) \geq 0$ and the boundary condition (24) imply the following:
(a) If $\beta\left(\varrho^{2}-\varphi^{2}\right) \geq 0$, then $\varphi \geq \max \left\{B_{1}, B_{2}\right\}$ or $\varphi \leq$ $\min \left\{B_{1}, B_{2}\right\}$.
(b) If $\beta\left(\varrho^{2}-\varphi^{2}\right) \leq 0$, then $\min \left\{B_{1}, B_{2}\right\} \leq \varphi \leq \max \left\{B_{1}, B_{2}\right\}$.

Below, we will present some implicit formulas for the single peak soliton solutions in the case of specific $\sigma$ and $\beta$.

Case $1\left((\sigma, \beta) \in A_{12}\right)$. In this case, we have $-\varrho<B_{2}<A<$ $B_{1}=\varrho$. From the standard phase analysis and Theorem 9 we know that if $\varphi$ is a single peak soliton of the $C(3,2,2)$ equation, then

$$
\varphi^{\prime}=-\sqrt{-\frac{\beta}{2}}(\varphi-A) \sqrt{\frac{\varphi-B_{2}}{\varphi+\varrho}} \operatorname{sgn}(\xi) .
$$

From the separation of variables we get

$$
\int h(\varphi) d \varphi=\sqrt{-\frac{\beta}{2}} \operatorname{sgn}(\xi) d \xi
$$

where $h(\varphi)=(1 /(A-\varphi)) \sqrt{(\varphi+\varrho) /\left(\varphi-B_{2}\right)}$. After a lengthy calculation of integral, we obtain the implicit solution $\varphi$ defined by

$$
H(\varphi):=\sqrt{\frac{A+\varrho}{A-B_{2}}} I_{1}(\varphi)-I_{2}(\varphi)=\sqrt{-\frac{\beta}{2}}|\xi|+K,
$$

where

$$
\begin{aligned}
& I_{1}(\varphi)=\ln \left|\frac{(A+\varrho)\left(\varphi-B_{2}\right)+\left(A-B_{2}\right)(\varphi+\varrho)+2 \sqrt{(A+\varrho)\left(A-B_{2}\right)\left(\varphi-B_{2}\right)(\varphi+\varrho)}}{\sqrt{(A+\varrho)\left(A-B_{2}\right)}(A+\varrho)(A-\varphi)}\right|, \\
& I_{2}(\varphi)=\ln \left|(\varphi+\varrho)+\left(\varphi-B_{2}\right)+2 \sqrt{(\varphi+\varrho)\left(\varphi-B_{2}\right)}\right|
\end{aligned}
$$

and $K$ is an arbitrary integration constant. For $\varphi(0)=\varrho$, the constant $K=H(\varphi(0))$ is defined by

$$
K=\sqrt{\frac{A+\varrho}{A-B_{2}}} I_{1}(\varrho)-I_{2}(\varrho)
$$

and for $\varphi(0)=B_{2}$,

$$
K=\sqrt{\frac{A+\varrho}{A-B_{2}}} I_{1}\left(B_{2}\right)-I_{2}\left(B_{2}\right) .
$$

(i) If $\varphi(0)=\varrho$, then $A<\varphi \leq \varrho$. Since $H^{\prime}(\varphi)=h(\varphi)$, we know that $H(\varphi)$ strictly decreases on the interval $(A, \varrho]$; thus, $H_{1}(\varphi)=\left.H\right|_{(A, \varrho]}(\varphi)$ gives a single peak soliton with $H_{1}(\varrho)=K$ and $H_{1}(A+)=\infty$. Therefore, $\varphi_{1}(\xi)=H_{1}^{-1}(\sqrt{-\beta / 2}|\xi|+K)$ is the solution satisfying

$$
\begin{aligned}
\varphi_{1}(0) & =\varrho, \\
\lim _{|\xi| \rightarrow \infty} \varphi_{1}(\xi) & =A, \\
\varphi_{1}^{\prime}(0 \pm) & = \pm(A-\varrho) \sqrt{\frac{\beta\left(B_{2}-\varrho\right)}{4 \varrho}} .
\end{aligned}
$$

So, $\varphi_{1}(\xi)$ is a peakon-like solution (see Figure 5).

(ii) If $\varphi(0)=B_{2}$, then $B_{2} \leq \varphi<A$. By $H^{\prime}(\varphi)=f(\varphi)$, we know that $H(\varphi)$ strictly increases on the interval $\left[B_{2}, A\right)$. Thus,

$$
H_{2}(\varphi)=\left.H\right|_{\left[B_{2}, A\right)}(\varphi)
$$




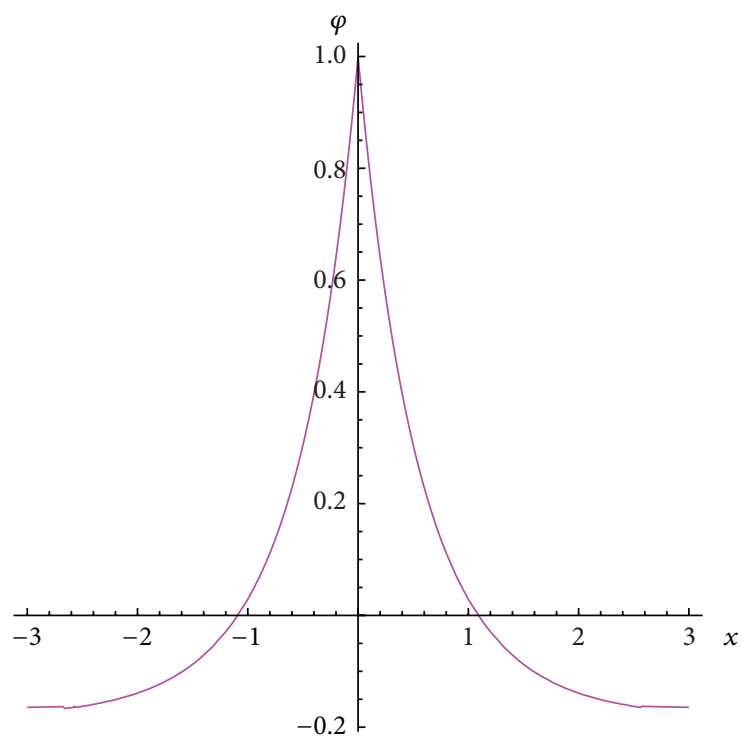

(a)

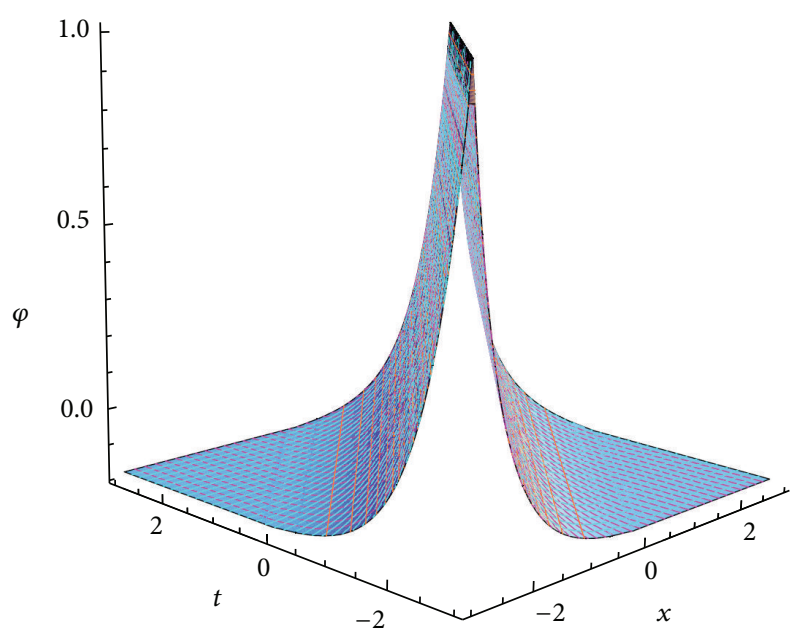

(b)

FIgURE 5: Two- and three-dimensional graphs of the peakon-like solution.

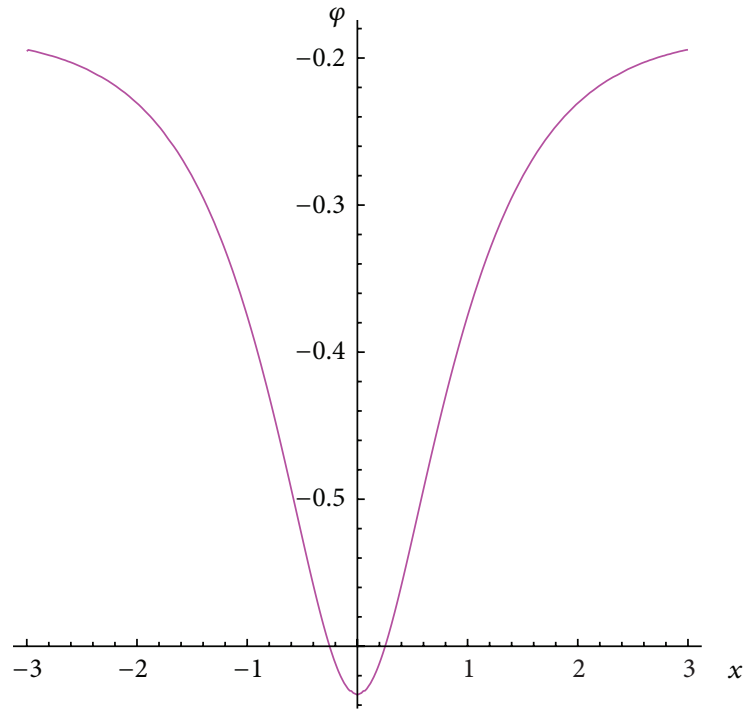

(a)

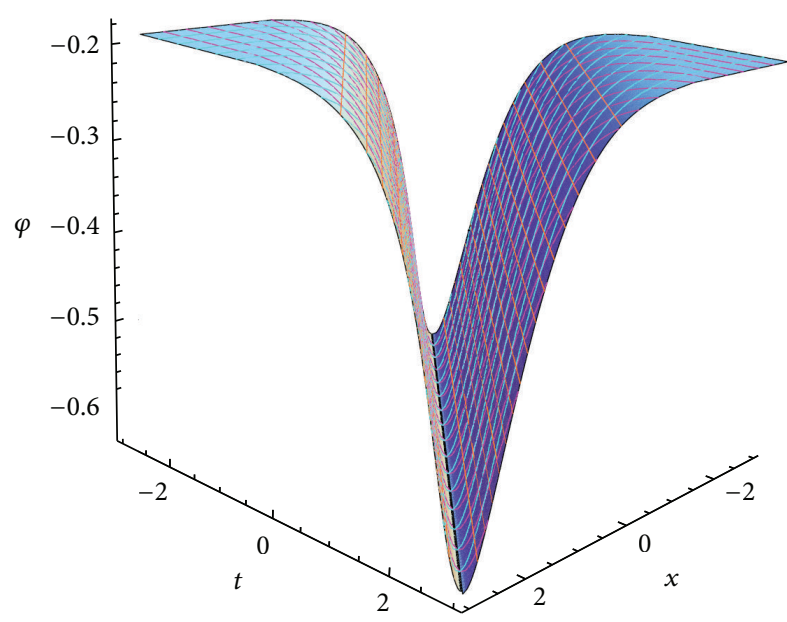

(b)

Figure 6: Two- and three-dimensional graphs of the smooth soliton solution.

has the inverse denoted by $\varphi_{2}(\xi)=H_{2}^{-1}(\sqrt{-\beta / 2}|\xi|+K)$. $\varphi_{2}(\xi)$ gives a kind of smooth soliton solution (see Figure 6) satisfying

$$
\begin{array}{r}
\varphi_{2}(0)=B_{2}, \\
\lim _{|\xi| \rightarrow \infty} \varphi_{2}(\xi)=A, \\
\varphi_{2}^{\prime}(0)=0 .
\end{array}
$$

Case $2\left((\sigma, \beta) \in A_{14}\right)$. In this case, we have $-\varrho=B_{2}<A<$ $\varrho<B_{1}$ and (25) is equivalent to

$$
\varphi^{\prime}=-\sqrt{-\frac{\beta}{2}}(\varphi-A) \sqrt{\frac{\varphi-B_{1}}{\varphi-\varrho}} \operatorname{sgn}(\xi) .
$$

Let

$$
g(\varphi)=\frac{1}{A-\varphi} \sqrt{\frac{\varphi-B_{1}}{\varphi-\varrho}}
$$




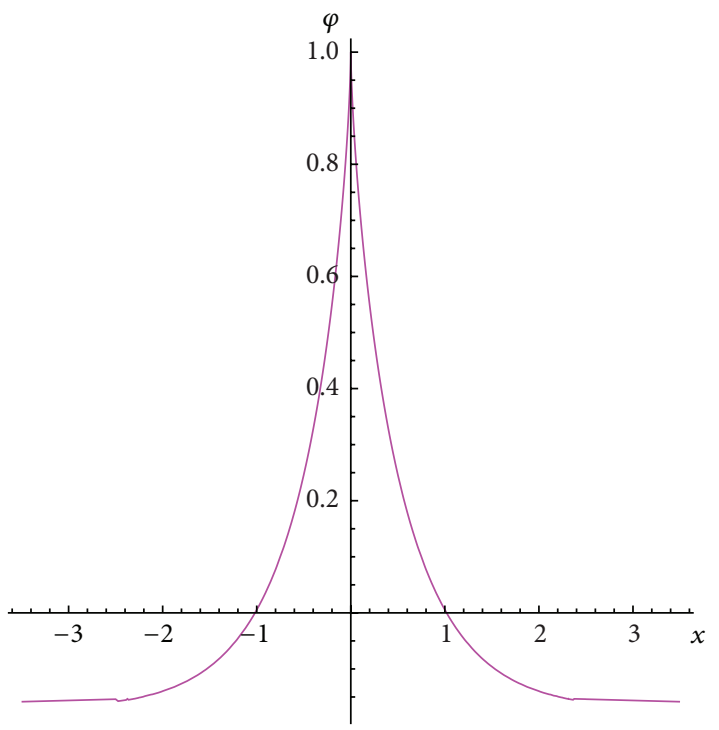

(a)

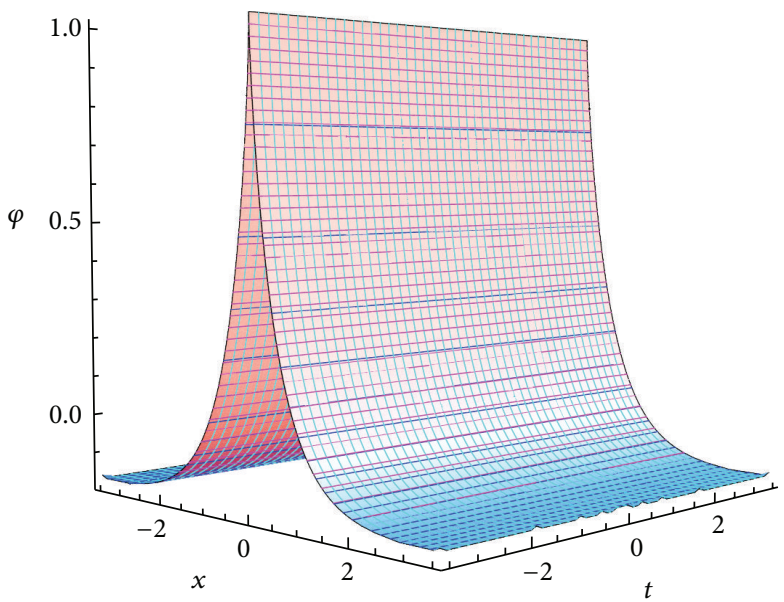

(b)

FIGURE 7: Two- and three-dimensional graphs of the cuspon solution.

then, (52) is converted to

$$
g(\varphi) d \varphi=\frac{1}{A-\varphi} \sqrt{\frac{\varphi-B_{1}}{\varphi-\varrho}} d \varphi=\sqrt{-\frac{\beta}{2}} \operatorname{sgn}(\xi) d \xi
$$

Integrating (54) on the interval $[0, \xi]$ (or $[\xi, 0])$ leads to the following implicit solutions:

$$
G(\varphi):=\sqrt{\frac{A-\varrho}{A-B_{1}}} I_{3}(\varphi)+I_{4}(\varphi)=\sqrt{-\frac{\beta}{2}}|\xi|+K,
$$

where

$$
\begin{aligned}
& I_{3}(\varphi)=\ln \left|\frac{(A-\varrho)\left(\varphi-B_{1}\right)+\left(A-B_{1}\right)(\varphi-\varrho)+2 \sqrt{(A-\varrho)\left(A-B_{1}\right)(\varphi-\varrho)\left(\varphi-B_{1}\right)}}{\sqrt{(A-\varrho)\left(A-B_{1}\right)}(A-\varrho)(\varphi-A)}\right|, \\
& I_{4}(\varphi)=\ln \left|(\varphi-\varrho)+\left(\varphi-B_{1}\right)+2 \sqrt{(\varphi-\varrho)\left(\varphi-B_{1}\right)}\right| .
\end{aligned}
$$

And $K$ is an arbitrary integration constant. It is obvious that, for $\varphi(0)=\varrho$, the constant $K=H(\varphi(0))$ is defined by

$$
K=\sqrt{\frac{A-\varrho}{A-B_{1}}} I_{3}(\varrho)+I_{4}(\varrho),
$$

and for $\varphi(0)=-\varrho$,

$$
K=\sqrt{\frac{A-\varrho}{A-B_{1}}} I_{3}(-\varrho)+I_{4}(-\varrho) .
$$

(i) If $\varphi(0)=\varrho$, then $A<\varphi \leq \varrho$. From $g(\varphi)<0$, we know that $G(\varphi)$ strictly decreases on the interval $(A, \varrho]$ with $G(\varrho)=K$ and $G(A+)=\infty$. Define

$$
G_{1}(\varphi)=\left.G\right|_{(A, \varrho]}(\varphi) .
$$

Since $G_{1}(\varphi)$ is a strictly decreasing function from $(A, \varrho]$ onto $[K, \infty)$, we can solve for $\varphi$ uniquely from (59) and obtain

$$
\varphi_{1}(\xi)=G_{1}^{-1}\left(\sqrt{-\frac{\beta}{2}}|\xi|+K\right) .
$$

It is easy to check that $\varphi$ satisfies

$$
\begin{aligned}
\varphi_{1}(0) & =\varrho, \\
\lim _{|\xi| \rightarrow \infty} \varphi_{1}(\xi) & =A, \\
\varphi_{1}^{\prime}(0 \pm) & =\mp \infty .
\end{aligned}
$$

Therefore, the solution $\varphi_{1}$ defined by (60) is a cuspon solution for the $C(3,2,2)$ equation (see Figure 7$)$. 
(ii) If $\varphi(0)=-\varrho$, then $-\varrho \leq \varphi<A$. Through a similar analysis, we get a strictly increasing function $G(\varphi)$ on the interval $[-\varrho, A)$ satisfying

$$
G(\varphi)=\sqrt{-\frac{\beta}{2}}|\xi|+K
$$

where $G(\varphi)$ is defined by (55). Let

$$
G_{2}(\varphi)=\left.G\right|_{[-\varrho, A)}(\varphi),
$$

then $G_{2}(\varphi)$ is a strictly increasing function from $[-\varrho, A)$ onto $[K, \infty)$ so that we can solve for $\varphi$ and obtain

$$
\varphi_{2}(\xi)=G_{2}^{-1}\left(\sqrt{-\frac{\beta}{2}}|\xi|+K\right) .
$$

It is easy to check that $\varphi_{2}$ satisfies

$$
\begin{aligned}
\varphi_{2}(0) & =-\varrho, \\
\lim _{|\xi| \rightarrow \infty} \varphi_{2}(\xi) & =A, \\
\varphi_{2}^{\prime}(0 \pm) & = \pm(A+\varrho) \sqrt{-\frac{\beta\left(B_{1}+\varrho\right)}{4 \varrho}} .
\end{aligned}
$$

Therefore, the solution $\varphi_{2}$ defined by (64) is a peakon-like solution, whose graph is similar to those in Figure 5.

Case $3\left((\sigma, \beta) \in A_{16}\right)$. In this case, we have $-\varrho<B_{2}<A=$ $0<B_{1}<\varrho, B_{2}=-B_{1}$, and

$$
\varphi^{\prime}=-\sqrt{-\frac{\beta}{2}} \varphi \sqrt{\frac{\varphi-B_{1}^{2}}{\varphi^{2}-\varrho^{2}}} \operatorname{sgn}(\xi)
$$

Hence from the separation of variables we have

$$
\frac{\sqrt{\varrho^{2}-\varphi^{2}}}{\varphi \sqrt{B_{1}^{2}-\varphi^{2}}} d \varphi=-\sqrt{-\frac{\beta}{2}} \operatorname{sgn}(\xi) d \xi
$$

Integrating (67) on the interval $[0, \xi]$ (or $[\xi, 0]$ ) leads to the following implicit formula for the two smooth soliton solutions:

$$
\begin{aligned}
& \frac{\sqrt{\varrho^{2}-\varphi^{2}}+\sqrt{B_{1}^{2}-\varphi^{2}}}{\sqrt{\varrho^{2}-B_{1}^{2}}}\left(\frac{\sqrt{\left(\varrho^{2}-B_{1}^{2}\right) \varphi^{2}}}{\varrho \sqrt{B_{1}^{2}-\varphi^{2}}+B_{1} \sqrt{\varrho^{2}-\varphi^{2}}}\right)^{\varrho} \\
& =\exp \left(-\sqrt{\left.-\frac{\beta}{2}|x-c t|\right)},\right.
\end{aligned}
$$

where $\varphi \in\left(A, B_{1}\right]$. Consider

$$
\begin{aligned}
& \frac{\sqrt{\varrho^{2}-B_{2}^{2}}}{\sqrt{\varrho^{2}-\varphi^{2}}+\sqrt{B_{2}^{2}-\varphi^{2}}}\left(\frac{\varrho \sqrt{B_{2}^{2}-\varphi^{2}}-B_{2} \sqrt{\varrho^{2}-\varphi^{2}}}{\sqrt{\left(\varrho^{2}-B_{2}^{2}\right) \varphi^{2}}}\right)^{\varrho} \\
& =\exp \left(\sqrt{\left.-\frac{\beta}{2}|x-c t|\right),}\right.
\end{aligned}
$$

where $\varphi \in\left[B_{2}, A\right)$.

Case $4\left((\sigma, \beta) \in A_{17}\right)$. In this case, we have $-\varrho=B_{2}<A=$ $0<B_{1}=\varrho$, and

$$
\varphi^{\prime}=-\sqrt{-\frac{\beta}{2}} \varphi \operatorname{sgn}(\xi)
$$

Choosing $\varphi(0)=\varrho($ or $-\varrho)$ as initial value, we get

$$
\begin{aligned}
& \int_{\varrho}^{\varphi} \frac{1}{\varphi} d \varphi=-\int_{0}^{\xi} \sqrt{-\frac{\beta}{2}} \operatorname{sgn}(\xi) d \xi, \text { for } A<\varphi \leq \varrho, \\
& \int_{-\varrho}^{\varphi} \frac{1}{\varphi} d \varphi=-\int_{0}^{\xi} \sqrt{-\frac{\beta}{2}} \operatorname{sgn}(\xi) d \xi, \text { for }-\varrho \leq \varphi<A,
\end{aligned}
$$

which immediately yield the peakon solutions

$$
\varphi(x-c t)= \pm \varrho \exp \left(-\sqrt{-\frac{\beta}{2}}|x-c t|\right) .
$$

The graphs for the peakon solution (72) are shown in Figure 8.

Remark 10. The classical peakon solution (72) and peakonlike solution (64) admit left-half derivative and right-half derivative at their crest. But the signs of the left-half derivative and right-half derivative are opposite, so the peakon and peakon-like solutions admit the discontinuous first order derivative at their crest. In comparison with classical peakon solution (72), the expression of the peakon-like solution (64) is more complex. Moreover, by observing Figures 2(14) and 2(17) we find that the phase orbits of the peakon consist of three straight lines, but the phase orbits of the peakon-like consist of two curves and a straight line. Therefore, we call the soliton solution (64) the peakon-like solution.

\section{Kink Wave and Kink Compacton Solutions}

We now turn our attention to the kink wave solutions of the $C(3,2,2)$ equation (10). In order to study kink wave solutions, we assume that

$$
\begin{aligned}
& \lim _{\xi \rightarrow \infty} \varphi(\xi)=A_{1}, \\
& \lim _{\xi \rightarrow-\infty} \varphi(\xi)=A_{2},
\end{aligned}
$$




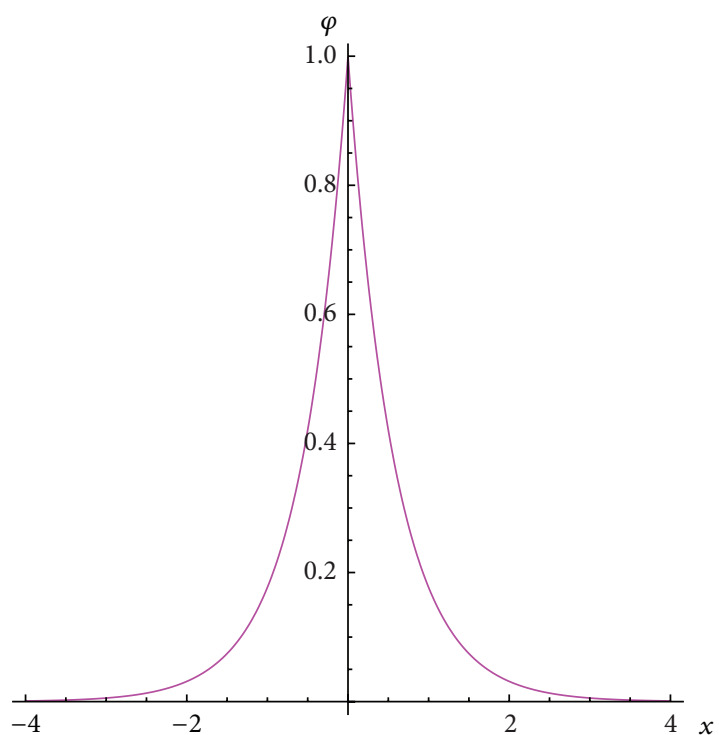

(a)

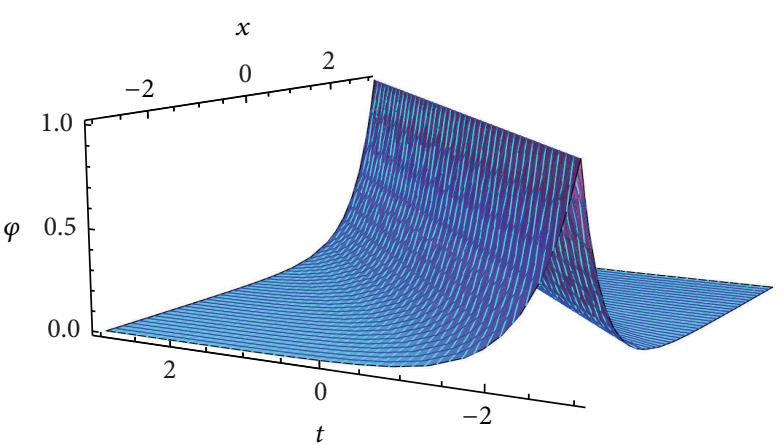

(b)

Figure 8: Two- and three-dimensional graphs of the peakon solution.

where $A_{1}>A_{2}$. Substituting the boundary condition (73)

into (14) generates

$$
\left(\varphi^{\prime}\right)^{2}=\frac{\beta\left(\varphi-A_{1}\right)\left(\varphi-A_{2}\right)\left[\varphi^{2}+\left(A_{1}+A_{2}\right) \varphi+A_{1}^{2}+A_{1} A_{2}+A_{2}^{2}+2 \epsilon / \beta\right]}{2\left(\varrho^{2}-\varphi^{2}\right)}:=F(\varphi) .
$$

The nonlinear differential equation (74) may sustain different kinds of nonlinear excitations. In what follows, we confine our attention to the cases $A_{2}=-A_{1}$ and $\epsilon=$ $-A_{1}^{2} \beta$ which describe kinks and kink compactons which play an important role in the dynamics systems. Under these considerations, (74) reduces to

$$
\left(\varphi^{\prime}\right)^{2}=\frac{\beta\left(\varphi-A_{1}\right)^{2}\left(\varphi-A_{2}\right)^{2}}{2\left(\varrho^{2}-\varphi^{2}\right)} .
$$

If $A_{1}<\varrho$, then from the phase analysis in Section 2 (see Figure $4(10))$, we know that $\left(A_{1}, 0\right)$ and $\left(A_{2}, 0\right)$ are two saddle points of (13) and the kink solutions can be obtained from the two heteroclinic orbits connecting $(\phi, y)=\left(A_{1}, 0\right)$ and $\left(A_{2}, 0\right)$. When $A_{1}$ increases upon reaching $\varrho$, that is $A_{1}=\varrho$, (75) becomes

$$
\left(\varphi^{\prime}\right)^{2}=-\frac{\beta\left(\varphi-A_{1}\right)\left(\varphi-A_{2}\right)}{2}
$$

and the ellipse $2 y^{2}+\beta\left(\varphi-A_{1}\right)\left(\varphi-A_{2}\right)=0$ (see Figure 4(11)), which is tangent to the singular lines $\varphi=\varrho$ and $\varphi=-\varrho$ at points $\left(A_{1}, 0\right)$ and $\left(A_{2}, 0\right)$, respectively, gives rise to two kink compactons of (10).

We next explore the qualitative behavior of kink wave solutions to (75) and (76). If $\varphi$ is a kink wave solutions of (75) or (76), we have $\varphi^{\prime} \rightarrow 0$ as $\varphi \rightarrow A_{1}$ and as $\varphi \rightarrow A_{2}$. Moreover, we have $F(\varphi) \geq 0$ for $A_{2} \leq \varphi \leq A_{1}$ and $\varphi$ is strictly monotonic in any interval where $F(\varphi)>0$. Thus, if $\varphi^{\prime}>0$ at some points, $\varphi$ will be strictly increasing until it gets close to the next zero of $F$. Denoting this zero $A_{1}$, we have $\varphi \uparrow A_{1}$. What will happen to the solution when it approaches $A_{1}$ ? Depending on whether the zero is double or simple, $\varphi$ has a different behavior. We explore the two cases in turn.

Theorem 11. (i) If $\varphi$ has a simple zero at $\varphi=A_{1}$, so that $F\left(A_{1}\right)=0$ and $F^{\prime}\left(A_{1}\right)<0$, then the solution $\varphi$ of (75) satisfies

$$
\varphi(\xi)-A_{1}=\frac{F^{\prime}\left(A_{1}\right)}{4}(\xi-\eta)^{2}+O\left((\xi-\eta)^{4}\right)
$$

as $\xi \uparrow \eta$,

where $\varphi(\eta)=A_{1}$.

(ii) When $\varphi$ approaches the double zero $A_{1}$ of $F(\varphi)$ so that $F^{\prime}\left(A_{1}\right)=0$ and $F^{\prime \prime}\left(A_{1}\right)>0$, then the solution $\varphi$ of $(75)$ satisfies

$$
\varphi(\xi)-A_{1} \sim \alpha \exp \left(-\xi \sqrt{F^{\prime \prime}\left(A_{1}\right)}\right) \quad \text { as } \xi \longrightarrow \infty
$$

for some constant $\alpha$. Thus, $\varphi \uparrow A_{1}$ exponentially as $\xi \rightarrow \infty$. 
Proof. (i) When $A_{1}=-A_{2}=\varrho$ and $\epsilon=-A_{1}^{2} \beta$, from (76), $F(\varphi)$ has a simple zero at $\varphi=A_{1}$. Then,

$$
\left(\varphi^{\prime}\right)^{2}=\left(\varphi-A_{1}\right) F^{\prime}\left(A_{1}\right)+O\left(\left(\varphi-A_{1}\right)^{2}\right)
$$

$$
\text { as } \varphi \uparrow A_{1} \text {. }
$$

Using the fact that $\left(\varphi^{\prime}\right)^{2} \geq 0$, we know that $F^{\prime}\left(A_{1}\right)<0$. Moreover,

$$
\frac{d \xi}{d \varphi}=\frac{1}{\sqrt{\left(\varphi-A_{1}\right) F^{\prime}\left(A_{1}\right)+O\left(\left(\varphi-A_{1}\right)^{2}\right)}} .
$$

Because

$$
\begin{aligned}
& \sqrt{\left(\varphi-A_{1}\right) F^{\prime}\left(A_{1}\right)+O\left(\left(\varphi-A_{1}\right)^{2}\right)} \\
& =\sqrt{A_{1}-\varphi}\left(\sqrt{-F^{\prime}\left(A_{1}\right)}+O\left(A_{1}-\varphi\right)\right), \\
& \frac{1}{\sqrt{-F^{\prime}\left(A_{1}\right)}+O\left(A_{1}-\varphi\right)} \\
& =\frac{1}{\sqrt{-F^{\prime}\left(A_{1}\right)}}+O\left(A_{1}-\varphi\right),
\end{aligned}
$$

we obtain

$$
\frac{d \xi}{d \varphi}=\frac{1}{\sqrt{-\left(A_{1}-\varphi\right) F^{\prime}\left(A_{1}\right)}}+O\left(\left(A_{1}-\varphi\right)^{1 / 2}\right) .
$$

Integrating (82) yields

$$
\eta-\xi=\frac{2}{\sqrt{-F^{\prime}\left(A_{1}\right)}}\left(A_{1}-\varphi\right)^{1 / 2}+O\left(\left(A_{1}-\varphi\right)^{3 / 2}\right),
$$

where $\eta$ satisfies $\varphi(\eta)=A_{1}$. Thus,

$$
(\eta-\xi)^{2}=\frac{4}{-F^{\prime}\left(A_{1}\right)}\left(A_{1}-\varphi\right)+O\left(\left(A_{1}-\varphi\right)^{2}\right),
$$

which implies $O\left(\left(A_{1}-\varphi\right)^{2}\right)=O\left((\eta-\xi)^{4}\right)$. Therefore, we get

$$
\varphi(\xi)=A_{1}+\frac{F^{\prime}\left(A_{1}\right)}{4}(\xi-\eta)^{2}+O\left((\xi-\eta)^{4}\right)
$$

as $\xi \uparrow \eta$,

where $\varphi(\eta)=A_{1}$.

(ii) When $A_{1}=-A_{2}<\varrho$ and $\epsilon=-A_{1}^{2} \beta$, from (75), $F(\varphi)$ has a double zero at $\varphi=A_{1}$. Then,

$$
\left(\varphi^{\prime}\right)^{2}=\left(\varphi-A_{1}\right)^{2} F^{\prime \prime}\left(A_{1}\right)+O\left(\left(\varphi-A_{1}\right)^{3}\right)
$$

as $\varphi \uparrow A_{1}$.

Furthermore, we get

$$
\frac{d \xi}{d \varphi}=\frac{1}{\sqrt{\left(\varphi-A_{1}\right)^{2} F^{\prime \prime}\left(A_{1}\right)+O\left(\left(\varphi-A_{1}\right)^{3}\right)}} .
$$

Observing that

$$
\begin{aligned}
& \sqrt{\left(\varphi-A_{1}\right)^{2} F^{\prime \prime}\left(A_{1}\right)+O\left(\left(\varphi-A_{1}\right)^{3}\right)} \\
& =\left(A_{1}-\varphi\right)\left(\sqrt{F^{\prime \prime}\left(A_{1}\right)}+O\left(A_{1}-\varphi\right)\right), \\
& \frac{1}{\sqrt{F^{\prime \prime}\left(A_{1}\right)}+O\left(A_{1}-\varphi\right)} \\
& =\frac{1}{\sqrt{F^{\prime \prime}\left(A_{1}\right)}}+O\left(A_{1}-\varphi\right),
\end{aligned}
$$

we obtain

$$
\frac{d \xi}{d \varphi}=\frac{1}{\left(A_{1}-\varphi\right) F^{\prime \prime}\left(A_{1}\right)}+O(1) .
$$

By a similar computation as the one that leads to (85), we arrive at (78). This completes the proof of Theorem 11.

Next we try to find the exact formulas for the kink wave solutions. Let $A_{1}=-A_{2}<\varrho$ and $\epsilon=-A_{1}^{2} \beta$. Then, (75) becomes

$$
\frac{\sqrt{\varrho^{2}-\varphi^{2}}}{\varphi^{2}-A_{1}^{2}} d \varphi=\mp \sqrt{\frac{\beta}{2}} d \xi .
$$

Integrating both sides of (90) gives the following implicit expressions of kink and antikink wave solutions:

$$
\begin{aligned}
& \arctan \frac{\varphi}{\sqrt{\varrho^{2}-\varphi^{2}}} \\
& \quad+\frac{\sqrt{\varrho^{2}-A_{1}^{2}}}{A_{1}} \operatorname{arctanh} \frac{\sqrt{\varrho^{2}-A_{1}^{2}}}{A_{1}} \frac{\varphi}{\sqrt{\varrho^{2}-\varphi^{2}}} \\
& = \pm \sqrt{\frac{\beta}{2} \xi .}
\end{aligned}
$$

By letting $A_{1} \rightarrow \varrho$ in (91), we get two kink compactons which are given by

$$
\pm \varphi= \begin{cases}\varrho \sin \sqrt{\frac{\beta}{2}} \xi, & |\xi| \leq \frac{\pi}{\sqrt{2 \beta}}, \\ \varrho, & \xi>\frac{\pi}{\sqrt{2 \beta}}, \\ -\varrho, & \xi<-\frac{\pi}{\sqrt{2 \beta}} .\end{cases}
$$

The graphs for the kink wave solutions (91) and kink compacton solutions (92) are shown in Figures 9 and 10, respectively.

Remark 12. The two kink compacton solutions (92) are different from the well-known smooth kink wave solutions. In comparison with kink wave solutions (91), the kink compacton solutions (92) have no exponential decay properties but have compact support. That is, they minus a constant, the differences identically vanish outside a finite core region. 


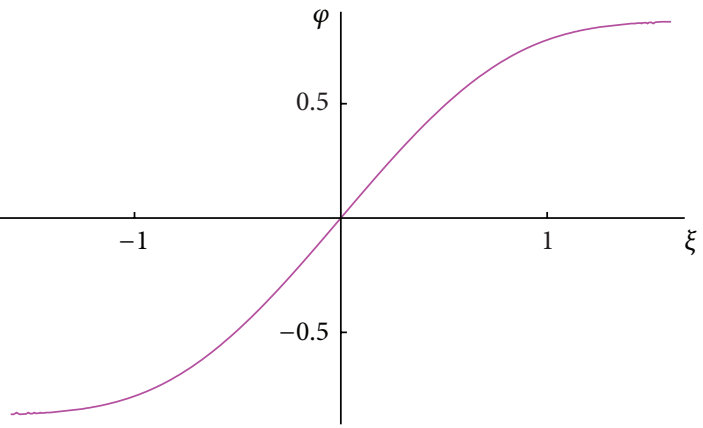

(a)

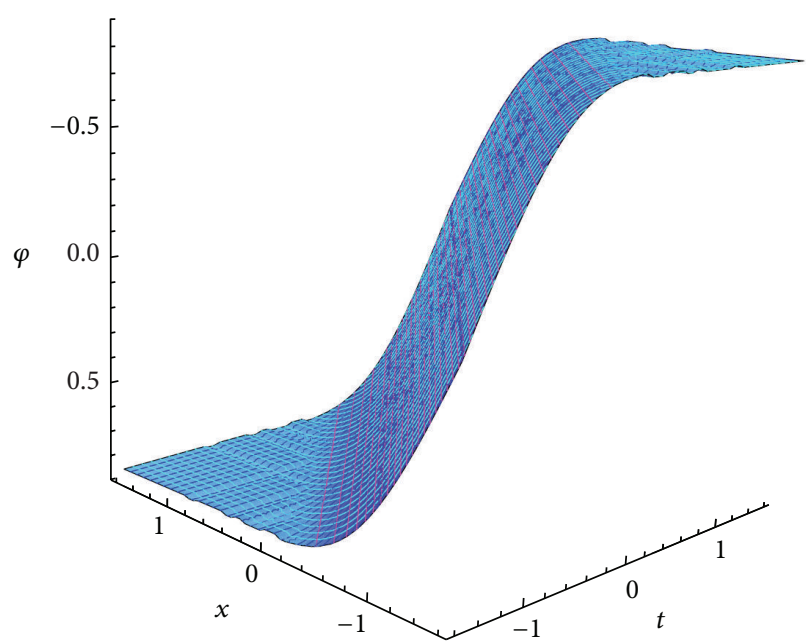

(b)

FIGURE 9: Two- and three-dimensional graphs of the kink wave solution.

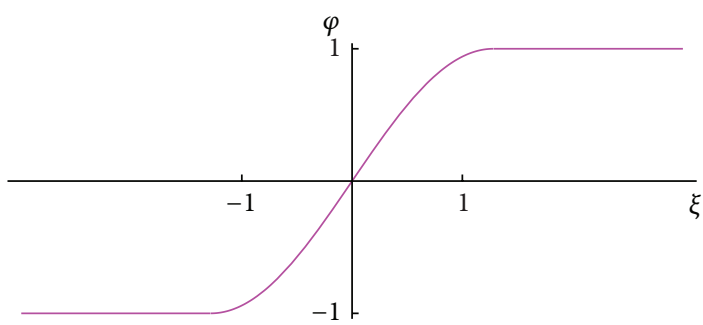

(a)

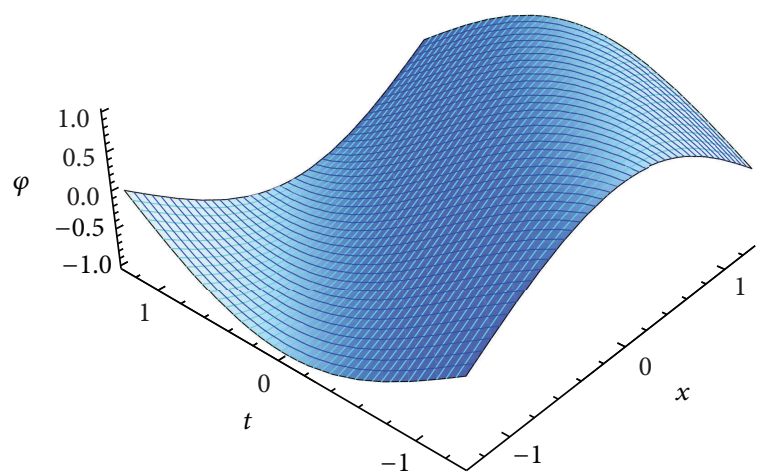

(b)

Figure 10: Two- and three-dimensional graphs of the kink compacton solution.

\section{Conclusion}

In this paper, we investigate the traveling wave solutions of the $C(3,2,2)$ equation $(10)$. We show that $(10)$ can be reduced to a planar polynomial differential system by transformation of variables. We treat the planar polynomial differential system by the dynamical systems theory and present a phase space analysis of their singular points. Two singular straight lines are found in the associated topological vector field. The influence of parameters as well as the singular lines on the smoothness property of the traveling wave solutions is explored in detail.

Because any traveling wave solution of $(10)$ is determined from Newton's equation which we write in the form $y^{2}=$ $F(\varphi)$, where $y=\varphi_{\xi}^{\prime}(\xi)$, we solve Newton's equation $y^{2}=$ $F(\varphi)$ for single peak soliton solutions and kink wave and kink compacton solutions. We classify all single peak soliton solutions of (10). Then peakon, peakon-like, cuspon, smooth soliton solutions of the generalized Camassa-Holm equation
(10) are obtained. The parametric conditions of existence of the single peak soliton solutions are given by using the phase portrait analytical technique. Asymptotic analysis and numerical simulations are provided for single peak soliton and kink wave and kink compacton solutions of the $C(3,2,2)$ equation.

Actually, for $m=2 k+1, k \in \mathbb{N}^{+}$in $C(m, 2,2)$ equation (9), the dynamical behavior of traveling wave solutions of (9) is similar to the case $m=3$; for $m=2 k, k \in \mathbb{N}^{+}$in $C(m, 2,2)$ equation (9), the dynamical behavior of traveling wave solutions of (9) is similar to the case $m=2$. We are applying the approach mentioned in this work to $C(2,2,2)$ equation (9) and already get some new solutions, which we will report in another paper.

\section{Conflict of Interests}

The authors declare that there is no conflict of interests regarding the publication of this paper. 


\section{Acknowledgments}

This work was partially supported by the National Natural Science Foundation of China (no. 11326131 and no. 61473332) and Zhejiang Provincial Natural Science Foundation of China under Grant nos. LQ14A010009 and LY13A010005.

\section{References}

[1] C. Rogers and W. R. Shadwick, Bäcklund Transformation and Their Applications, Academic Press, New York, NY, USA, 1982.

[2] W. Malfliet and W. Hereman, "The tanh method: I. Exact solutions of nonlinear evolution and wave equations," Physica Scripta, vol. 54, no. 6, pp. 563-568, 1996.

[3] R. Hirota, Direct Method in Soliton Theory, Springer, Berlin, Germany, 1980.

[4] J. S. Cohen, Computer Algebra and Symbolic Computation: Mathematical Methods, A K Peters, 2003.

[5] S. Lie, "Uber die integration durch bestimmte integrale von einer klasse linearer partieller differential eichungen," Archiv der Mathematik, vol. 6, pp. 328-368, 1881.

[6] H.-H. Dai and X.-H. Zhao, "Traveling waves in a rod composed of a modified Mooney-Rivlin material I: bifurcation of equilibra and non-singular case," Proceedings of the Royal Society A: Mathematical, Physical and Engineering Sciences, vol. 455, no. 1990, pp. 3845-3874, 1999.

[7] H.-H. Dai and F.-F. Wang, "Asymptotic bifurcation solutions for compressions of a clamped nonlinearly elastic rectangle: transition region and barrelling to a corner-like profile," SIAM Journal on Applied Mathematics, vol. 70, no. 7, pp. 2673-2692, 2010.

[8] J. Li and Z. Liu, "Smooth and non-smooth traveling waves in a nonlinearly dispersive equation," Applied Mathematical Modelling, vol. 25, no. 1, pp. 41-56, 2000.

[9] A. Chen and J. Li, "Single peak solitary wave solutions for the osmosis $\mathrm{K}(2,2)$ equation under inhomogeneous boundary condition," Journal of Mathematical Analysis and Applications, vol. 369, no. 2, pp. 758-766, 2010.

[10] R. Camassa and D. D. Holm, "An integrable shallow water equation with peaked solitons," Physical Review Letters, vol. 71, no. 11, pp. 1661-1664, 1993.

[11] P. Rosenau, "On nonanalytic solitary waves formed by a nonlinear dispersion,” Physics Letters A, vol. 230, no. 5-6, pp. 305-318, 1997.

[12] P. Rosenau and J. M. Hyman, "Compactons: solitons with finite wavelength," Physical Review Letters, vol. 70, no. 5, pp. 564-567, 1993.

[13] L. Zhang, L.-Q. Chen, and X. Huo, “The effects of horizontal singular straight line in a generalized nonlinear Klein-Gordon model equation," Nonlinear Dynamics, vol. 72, no. 4, pp. 789801, 2013.

[14] J. Li and H. Dai, On the Study of Singular Nonlinear Traveling Wave Equations: Dynamical System Approach, Science Press, Beijing, China, 2007.

[15] A. Chen, S. Wen, S. Tang, W. Huang, and Z. Qiao, "Effects of quadratic singular curves in integrable equations," Studies in Applied Mathematics, vol. 134, no. 1, pp. 24-61, 2015.

[16] L. Tian and J. Yin, "New compacton solutions and solitary wave solutions of fully nonlinear generalized Camassa-Holm equations," Chaos, Solitons \& Fractals, vol. 20, no. 2, pp. 289-299, 2004.
[17] L. Zhang, "The bifurcation and peakons for the special $C(3,2,2)$ equation," Pramana, vol. 83, no. 3, pp. 331-340, 2014.

[18] J. Lenells, "Traveling wave solutions of the Camassa-Holm equation," Journal of Differential Equations, vol. 217, no. 2, pp. 393-430, 2005.

[19] Z. J. Qiao and G. P. Zhang, "On peaked and smooth solitons for the Camassa-Holm equation," Europhysics Letters, vol. 73, no. 5, pp. 657-663, 2006. 


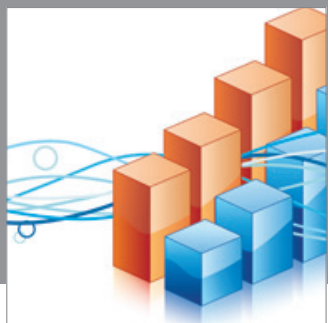

Advances in

Operations Research

mansans

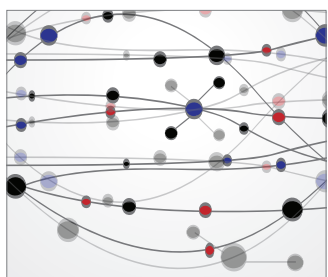

The Scientific World Journal
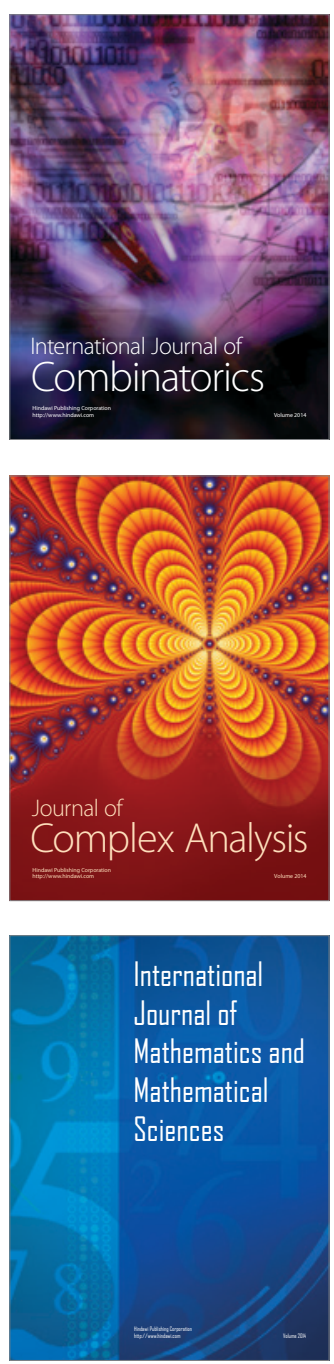
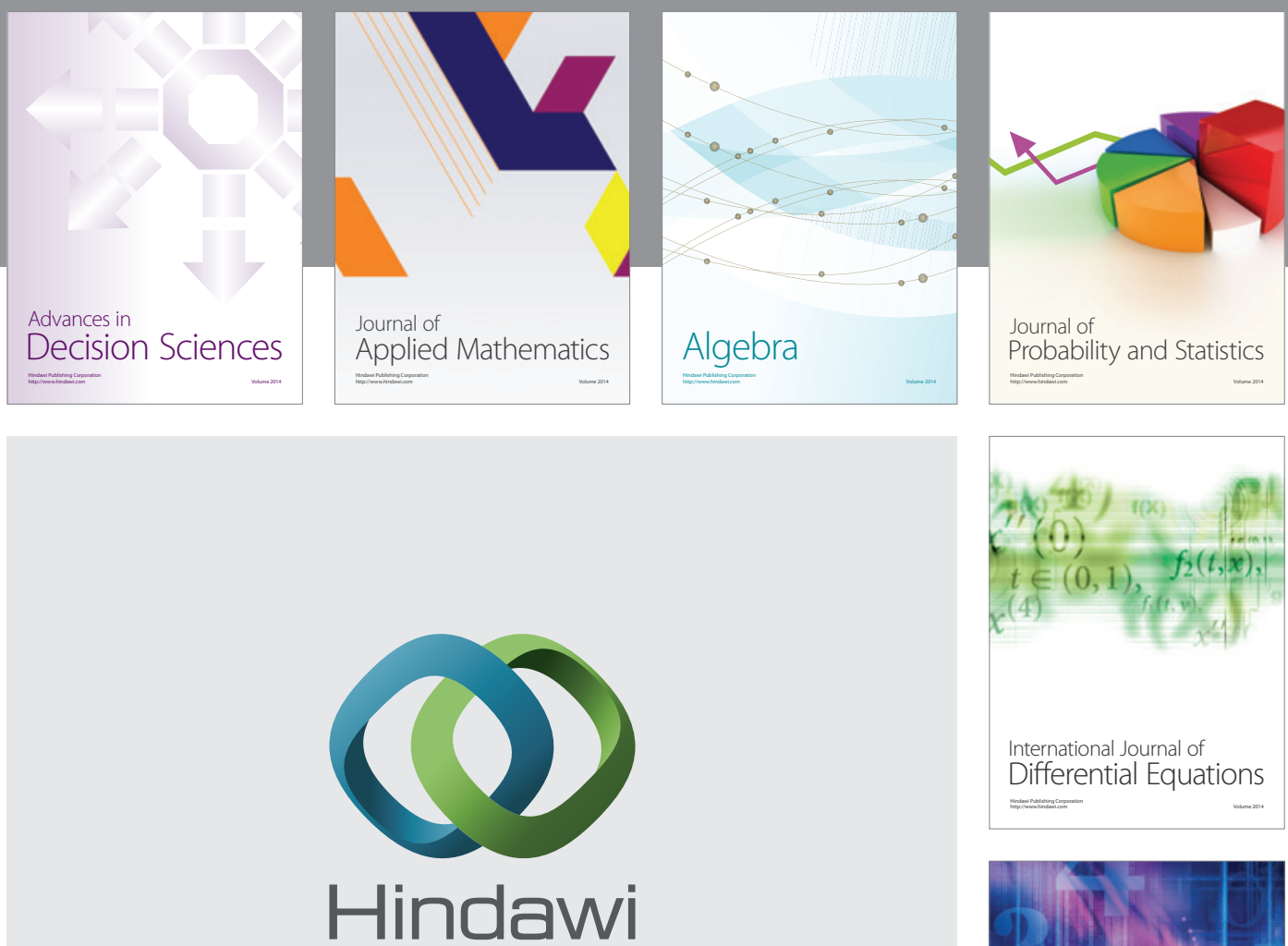

Submit your manuscripts at http://www.hindawi.com
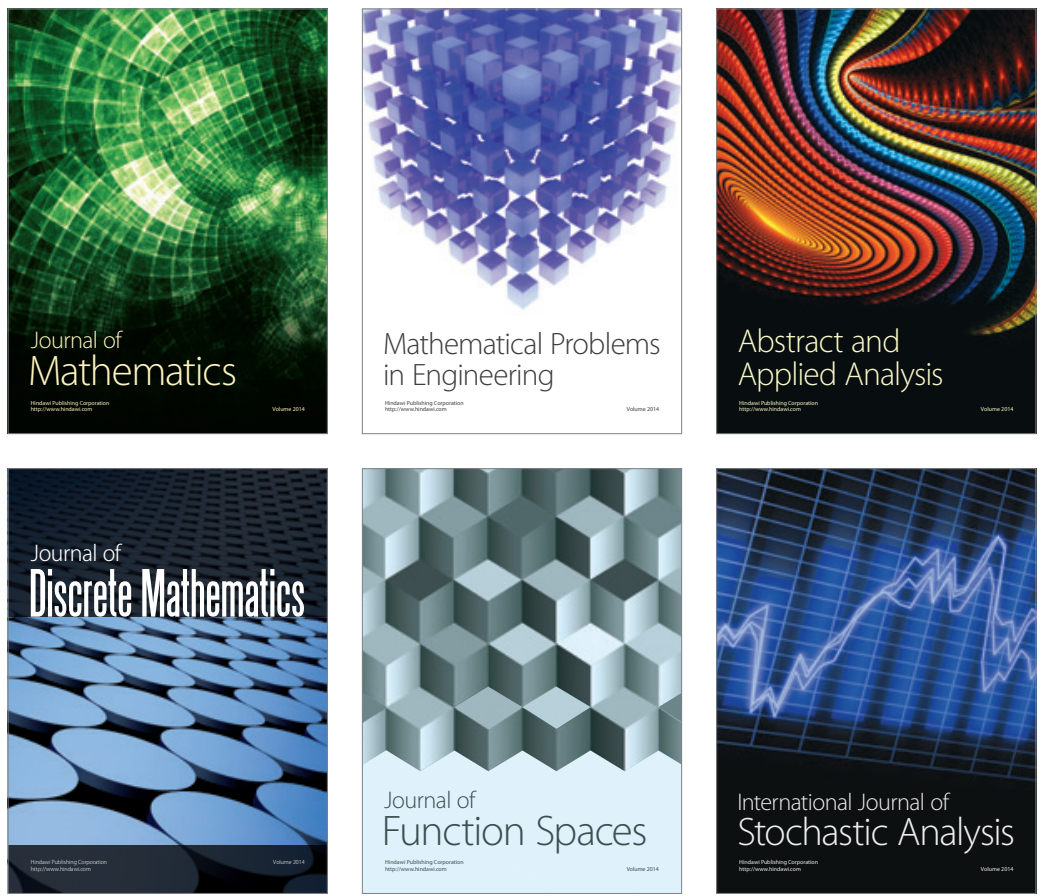

Journal of

Function Spaces

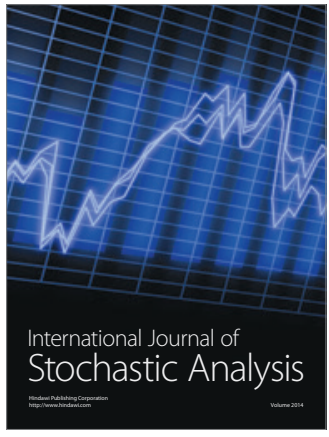

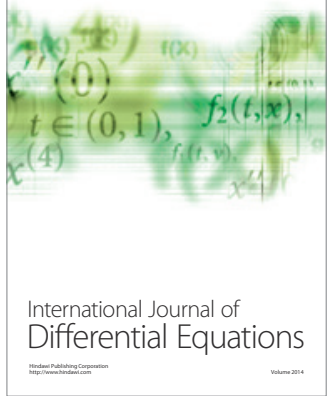
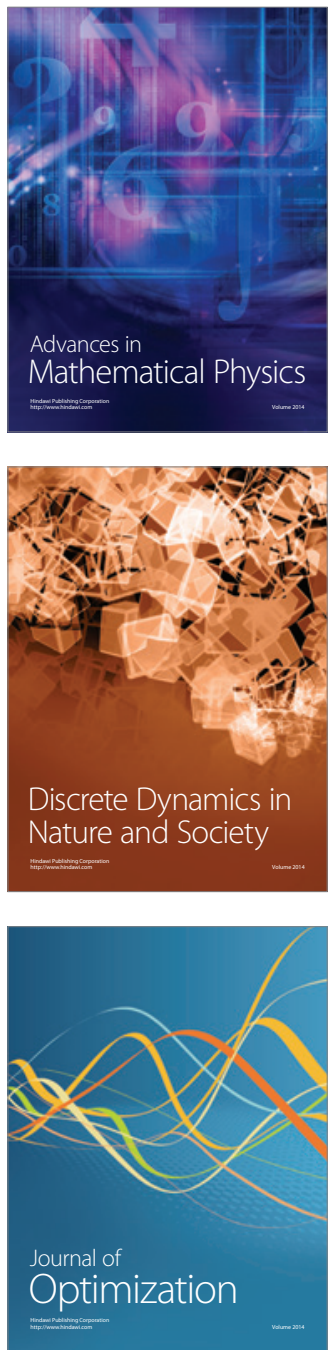